\title{
Intermodulation Distortion Analysis of a Guitar Distortion Pedal With a Starving Circuit
}

\author{
MASAKI INUI, ${ }^{1}$ AES Student Member, \\ (da20001@cc.it-hiroshima.ac.jp) \\ TOSHIHIKO HAMASAKI, ${ }^{1}$ AES Member AND MENNO VAN DER VEEN, ${ }^{2}$ AES Member \\ (t.hamasaki.rs@it-hiroshima.ac.jp) \\ (info@mennovanderveen.nl) \\ ${ }^{1}$ Information Systems and Science, Graduate School of Science and Technology, Hiroshima Institute of Technology, \\ Hiroshima, 731-5193, Japan \\ ${ }^{2}$ Ir. Bureau Vanderveen, $8743 \mathrm{KZ}$ 18, Hichtum, The Netherlands
}

\begin{abstract}
Despite the recent trend of digital transformation in the music industry, the popularity of guitar effects pedals (GEPs) designed with analog components has not declined. This paper describes the complexity of the nonlinear characteristics of the analog circuitry in a distortion pedal, which originates not only from clipping diodes but also from the integrated operational amplifier itself. It is well known that variation in the supply voltage of a distortion pedal influences its sound. Based on this phenomenon, we have designed a voltage-starving circuit to control various distorted transfer functions depending on the frequency. Particular attention is given to the difference between odd and even nonlinearity in the mechanism of generating intermodulation distortion (IMD) for two-tone dissonance and consonance. These transfer functions are analyzed in detail using a 9th-order polynomial approximation. As a result, all peaks of the intermodulation frequencies are successfully identified in a complex spectrum. Furthermore the spectral shape of the measured IMD peaks is reproduced with an error of less than $50 \mathrm{~dB}$ by the simulation of their approximate formula.
\end{abstract}

\section{O INTRODUCTION}

The main objective of this study is to investigate the variation in the even-order harmonics of 'distortion' pedals. Basically, the harmonics of the distorted sound originate from the circuit topology as well as physical characteristics of the active and passive devices, such as the semiconductor materials used. In the mid-to-late 1960s, the first circuits for guitar effects pedals (GEPs) were developed in the form of fuzz circuits, such as "Tone Bender MKI" and "Fuzz Face" in 1965 and 1966, respectively, and in the form of frequency booster "Rangemaster" in 1966. A germanium PNP bipolar junction transistor (BJT) was implemented as a core device for both types of circuits. A fuzz circuit is similar to a common-emitter two-stage high-gain amplifier, but it includes feedback from the emitter of the second transistor to the input base terminal of the first transistor via a resistor. The feedback voltage is controlled by a frequency- dependent variable emitter resistance. As a result of this feedback, the output wave shape becomes rich in asymmetric even-order harmonics, with a tall rectangular pulse. In a frequency booster, the bias of the input node of the PNP BJT is shifted by a voltage divider below the middle point of the supply voltage rail, which creates an asymmetric-shaped output wave shape. In a later product of "Zonk Machine II," the PNP BJTs were replaced with silicon. Because of the simplicity of the circuit diagrams for these products, it can be expected that their sound characteristics will be sensitive to manufacturing deviations, especially with regard to the properties of the BJTs, which can be represented by a family of curves of the collector current vs. the emittercollector voltage. Additionally the supply voltage rejection ratio is likely to be quite low in these circuits; therefore the voltage starvation technique will be very effective.

While fuzz and frequency booster circuits were undergoing a series of modifications, the first series of operational 
amplifiers (OPAs) was developed in the same decade, the famous series of $\mu \mathrm{A} 702,709,723$, and 741 , and then in 1974, the first OPA-based distortion pedal "M104, Distortion +" came onto the market. This distortion pedal consisted of a noninverting OPA, a clipping circuit with antiparallel germanium diodes, and a resistor-capacitor (RC) low-pass filter. Later, in the 1980s, an overdrive pedal "Tube Screamer, TS808" was developed by inserting silicon antiparallel diodes into the OPA feedback circuit, enabling the generation of a symmetric output, equal to the first OPA-based distortion pedal "M104, Distortion +".

Following this product, an asymmetric combination of 3 antiparallel diodes ( 2 serially connected silicon diodes on one side and 1 inversely connected silicon diode) in the feedback of the OPA was proposed by another manufacturer, named "Super Overdrive SD-1." The idea of this connection was to obtain an asymmetric output waveform containing even harmonics, resulting in a significant sound change compared with the previous design [1]. Due to the difference in threshold voltage between silicon and germanium $(0.7 \mathrm{~V}$ or more for $\mathrm{Si}$ compared to $0.3 \mathrm{~V}$ or less for $\mathrm{Ge}$ ), the degree of asymmetry can be modified by replacing 1 or more silicon diodes with germanium, i.e., using combinations such as 2 silicon diodes +1 germanium diode or 2 germanium diodes +1 silicon diode. Based on this idea, the sound differences of overdrive pedals depending on even harmonics have been studied in psychoacoustic tests [2] considering various predictors of perception [3,4]. In a broad sense, the overdrive mode is recognized as one kind of distortion mode. Therefore, in this article, we will consider the term 'distortion pedal' to also encompass the concept of 'overdrive pedal.'

In current audio systems, the purpose of using an OPA is to obtain linear amplification characteristics by applying a feedback circuit. Therefore the supply voltage for an OPA in a hi-fi audio system is typically chosen to be as high as $+/-15 \mathrm{~V}$ [5]. However, when an OPA is used in a distortion or fuzz pedal, its role is essentially to increase the amplification from a guitar pickup, and furthermore, portability is a key requirement; therefore the supply voltage is provided by a $9 \mathrm{~V}$ dry cell. It is well known by guitarists that the distorted sound changes as the dry cell nears the end of its life and the voltage begins to drop. This fact has led to the conscious use of the voltage starvation technique $[6,7]$ and remains a topic of interest on related websites [8].

The key means of digitizing such pedals is through modeling of the corresponding analog circuit blocks. The realtime processing of some of the passive components has currently been achieved due to the simplicity of the physical model. Additionally the saturation of nonlinearities by means of clipping diodes in a distortion pedal can be approximated by hyperbolic functions $[9,10]$. On the other hand, because of reactive factors, the digital implementation of active devices has not been completely addressed. The major approach has been to use a small-signal equivalent circuit model that directly simulates the circuit behavior [11]. Recently the wave digital filter technique [12] has been extended to analog circuit blocks $[13,14]$. Additionally success has been achieved in the large-signal modeling of the active circuit blocks in a frequency booster pedal. However the OPA function was implemented as an ideal model with no nonlinear dynamic characteristics [15].

The situation is similar for analog synthesizers, such as in the "West Coast" tradition of analog sound synthesis, which relies on wavefolders for sound generation. The core of a wavefolder circuit consists of an NPN BJT and a PNP BJT, which can be digitized by means of large-signal equivalent circuits [16]. It should be noted that these "virtual analog" models have been achieved by assuming symmetric nonlinear (i.e., with odd-order harmonics) transfer functions. On the other hand a black box approach based on deep learning has been proposed for the real-time processing of nonlinear transfer characteristics [17,18]. Although these latest methods are considered to be among the most useful tools for advancing digitization, certain aspects of their applicability, such as the reproducibility of harmonics for complex analog signal processing modes, are yet unknown.

At the AES convention in 2017, the authors' group presented the ability to control the even-order harmonics of an OPA-based distortion pedal by means of voltage starvation associated with the gain [19]. The next year we demonstrated a supply-voltage-starving control circuit with a DC/DC converter for searching for the effective voltage range for sound changes due to intermodulation distortion (IMD) [20]. However, a means of simultaneously achieving the virtual analog modeling of both odd-order and evenorder harmonics has not yet been found. The unsolved problems include the complexity of the large-signal nonlinear behavior arising from the complexity of the starved OPA.

Therefore the purposes of this research are as follows. First, we wish to clarify the cause of the starvation effect in the OPA and achieve stable reproduction thereof by reverse engineering the internal circuitry of the OPA. Second, we wish to characterize the dependence of both the odd-order and even-order distortion on the starvation voltage in a prototype distortion pedal and obtain a mathematical model that is relatively easy to digitally process to describe the transfer characteristics. Third, we wish to determine the error tolerance of the model simulations for IMD, for which the differences in the distortion components are more audible than those in a single-tone case, under various settings of the distortion pedal. Fourth, we believe that the distortion pedal created in this study will facilitate more quantitative discussion and validation of the results of statistical psychoacoustic tests for sounds rich in even-order harmonics, as discussed in reference [2].

This paper is organized as follows. In Sec. 1, we describe the mechanism of even-harmonic generation using the fundamental distortion circuitry associated with simplified schematics of an OPA. The system configuration of the developed distortion pedal, including the power management circuitry for voltage starvation, is described in Sec. 2. In Sec. 3, the experimental setups and measurement conditions are introduced. The fundamental response of the distortion pedal is presented in Sec. 4, including the starvation effect. In Sec. 5, the transfer characteristics of a onetone signal under various distortion conditions are demonstrated by comparing measurements and simulations with a 
polynomial approximation as a reference. Subsequently, the intermodulation spectra of two-tone signals are discussed in detail in Sec. 6. Finally, Sec. 7 presents the conclusion and discusses future research.

\section{MECHANISM OF NONLINEAR OPA OPERATION IN A DISTORTION PEDAL}

We studied the circuit diagrams of more than 40 distortion and overdrive pedals, including those listed in Dave Hunter's handbook [21]. OPAs with noninverting connections are utilized in this sound category; the NJM4558 is especially popular [22]. To clarify the generation mechanisms of even-harmonic and odd-harmonic distortion, we evaluated a fundamental circuit consisting of a high-gain noninverting OPA (NJM4558), antiparallel clipping diodes, and an RC filter in series, as shown in Fig. 1.

As shown in the inset photograph on the left-hand side, an asymmetric (even-harmonic) distortion of the plus/minus period is observed in the pulsed waveform at the output of the OPA, which is clipped to $4.37 \mathrm{~V}_{\mathrm{p}-\mathrm{p}}$ for a $200 \mathrm{mV}_{\mathrm{p}-\mathrm{p}}$ input with a $9 \mathrm{~V}$ power supply. The pulsed signal is clipped again to $1.07 \mathrm{~V}_{\mathrm{p}-\mathrm{p}}$ by the bidirectional diodes while maintaining the asymmetry ratio, as shown in the photograph on the right-hand side. This observation motivated the research presented in this paper on the development of a system for controlling both even and odd harmonics with voltage starvation [19].

To confirm that this phenomenon can be stably reproduced, the change in the total current of the OPA with respect to the voltage starvation was measured. Ten OPAs in total were measured, which were obtained through different sales channels (A, B and C) and stamped with different manufacturing lot numbers. The supply voltage $\mathrm{V}_{\mathrm{cc}}$ was varied from $0 \mathrm{~V}$ to $9 \mathrm{~V}$ in $0.1 \mathrm{~V}$ increments, from $9 \mathrm{~V}$ to $12 \mathrm{~V}$ in $0.4 \mathrm{~V}$ increments, and then down to $0 \mathrm{~V}$ in the same voltage increments as in the upward case in each supply voltage range. Additionally the measurements were performed in an atmospheric temperature range of 25 to 85 degrees centigrade in the same operating state as the system depicted in Fig. 1.

As shown in Fig. 2, the total consumed current $\mathrm{I}_{\text {total }}$ is almost flat in the product specification range $\left(8 \mathrm{~V}<\mathrm{V}_{\mathrm{cc}}\right)$, although it shows some deviation among the different groups of lot numbers. For each device, the current $\mathrm{I}_{\text {total }}$ suddenly begins to drop with decreasing $\mathrm{V}_{\mathrm{cc}}$, following Ohm's law, at the knee point of $6.8 \mathrm{~V}$. Then the current abruptly decreases rapidly at a $\mathrm{V}_{\mathrm{cc}}$ of $2 \mathrm{~V}$. In general, the reference voltage for the current source circuit in a silicon integrated circuit is provided by a Zener diode. The threshold voltage of a silicon PN-junction Zener diode is approximately $7 \mathrm{~V}$; accordingly, it is highly possible that such a Zener diode is installed at the right end of the circuit diagram (shown in Fig. 3 [22]) to serve as a reference for the current source. The ohmic region, $\mathrm{V}_{\mathrm{cc}} 2 \mathrm{~V}$ to $6.8 \mathrm{~V}$, originates from resistor R9. The operational stability of these circuits is typically checked in a temperature range of up to 75 degrees centigrade, as shown in Fig. 2(b), to ensure consumer product quality.
As shown in the figure, the total current decreases slightly with increasing temperature. However there is no significant operational deterioration due to temperature. Thus the supply voltage starving of the OPA can be qualified from the viewpoint of functional reliability.

Therefore the phenomena observed in Fig. 1 should be attributable to the overdrive mechanism of the noninverting OPA connection. Based on reverse engineering of the circuit diagram shown in Fig. 3, the mechanism of the voltagestarving effect can be explained as follows:

The OPA is designed with integrated silicon BJT technology and consists of four stages, as shown by the simplified circuit diagram in Fig. 3.

$1^{\text {st }} \quad$ Differential amplification Q1,2
$2^{\text {nd }} \quad$ Emitter-follower buffer Q5
$3^{\text {rd }} \quad$ Common-Emitter high-gain amplification Q6
$4^{\text {th }} \quad$ Push-pull class AB output amplification
Q7,.., 10 (current sources are not listed)

The essential point to note is the inherent asymmetry in the third-stage circuit. This is not a problem at linear signal levels for general applications. However, if the feedback gain is increased to intentionally introduce distortion, this causes asymmetry at the output. The third amplification stage, with the NPN transistor $\mathrm{Q}_{6}$, is designed to have a high gain with the active-load NPN transistor Q12, where the biasing circuit for the next stage is merged. The diodeequivalent connection of NPN transistors Q7 and Q8 with R6 provides the emitter-base bias for NPN transistor Q9 and PNP transistor Q10 for the final push-pull class AB amplification stage. Therefore the output signal $v_{\text {cq6 }}$ of NPN Q6 for the high-gain setting of the noninverting OPA is clipped asymmetrically because of the headroom reduction achieved by this complicated connection of components.

Even in the virtual ground principle of the OPA for $\mathrm{V}+$ and $\mathrm{V}-$, the asymmetry between the sinusoidal signal input at the $\mathrm{V}+$ pin and the clipped signal feedback input at the $\mathrm{V}-$ pin in the first differential stage is enhanced by the nonlinear properties of the components, such as the parasitic capacitance of the input PNP transistors Q1 and Q2 and the biasing NPN transistor Q11. The distorted output voltage $v_{\text {cq2 }}$ of Q2 is buffered by the second NPN transistor Q5 in the emitter-follower stage when proceeding toward the third stage. Furthermore, as mentioned in SEC. 0, the power supply voltage of the pedal $\mathrm{V}_{\mathrm{cc}}=9 \mathrm{~V}(+/-4.5 \mathrm{~V})$ is significantly lower than the $30 \mathrm{~V}(+/-15 \mathrm{~V})$ used for hi-fi applications. As a result the asymmetry of the OPA output swing is enlarged relative to the headroom of the supply voltage, thus making the voltage starvation technique effective.

\section{CIRCUIT DESIGN OF A STARVATION-CONTROLLED DISTORTION PEDAL}

Fig. 4 shows the circuit schematics of the newly developed distortion pedal. The signal processing chain, which consists of three stages, is shown in (a), and the schematic for the power management section is shown in (b). The core 


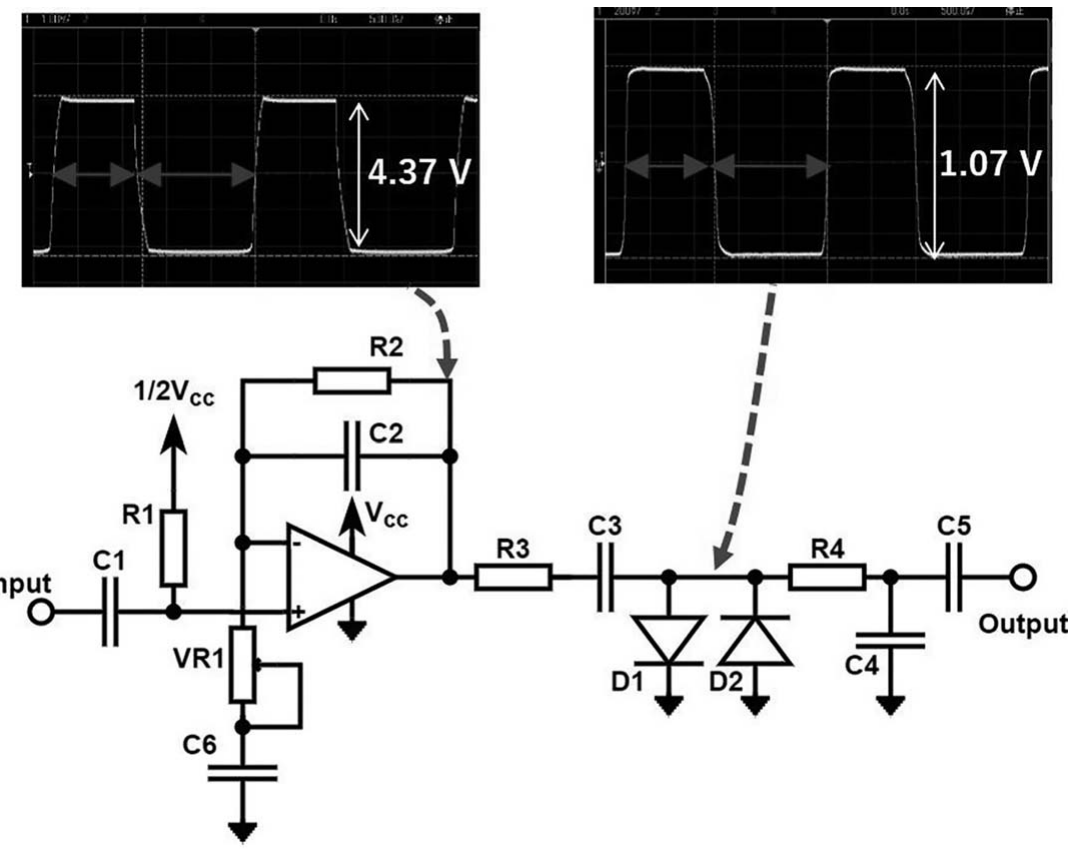

Fig. 1. Waveforms of a high-gain OPA output and a clipped node by antiparallel diodes in an "overdrive" pedal. The input signal is $200 \mathrm{mV}_{\mathrm{p}-\mathrm{p}}, 441 \mathrm{~Hz}$.

of the signal processing chain is a distortion generation circuit, consisting of OPA2 and a couple of diodes along with a $1^{\text {st }}$-order RC low-pass filter. The original gain of OPA2 and the RC constant of the filter are designed to generate a sound effect categorized as overdrive. Voltage starving is applied only at OPA2, while the supply voltage of the OPA 1 and OPA3 buffers is maintained at $12 \mathrm{~V}$ to ensure linear operation based on the data sheet. We have discovered that the input impedance of the OPA2 gains feedback circuitry depending on the starved voltage. To prevent sound modulation caused by changes in the input impedance, we add OPA1 as a high-impedance input buffer. This is an im- provement to the previous version of this distortion pedal presented at the $144^{\text {th }}$ AES convention [20].

One additional improvement from the previous circuit is that we have modified the reference voltage circuitry of the NJM2392 DC/DC converter to widen the tuning range of the audible difference. The ripple noise in the power line from the $\mathrm{AC}$ adaptor is filtered out by an $\mathrm{RC}$ lowpass filter, which consists of a BJT capacitance multiplier circuit. The power for the $1^{\text {st }}$-stage buffer OPA1 and the output buffer OPA3 is supplied directly from this output to avoid additional distortion and noise.

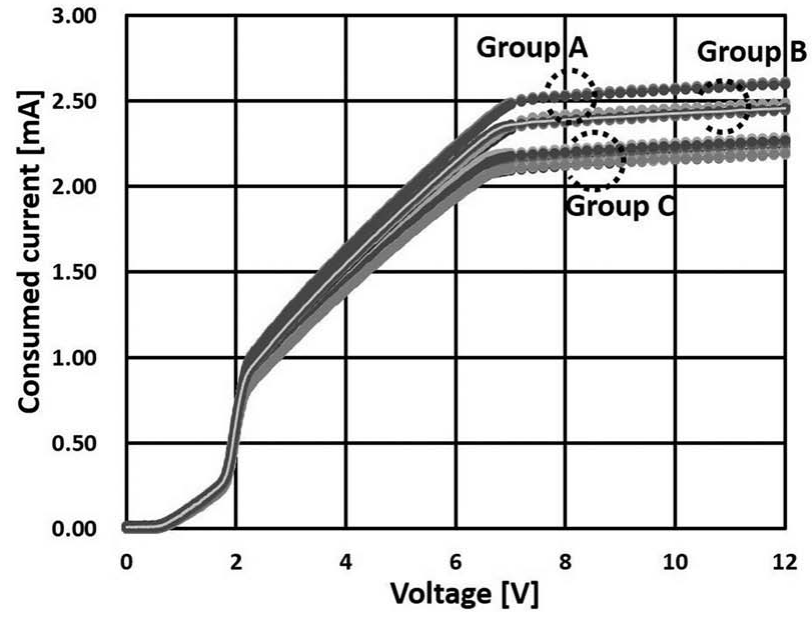

(a)

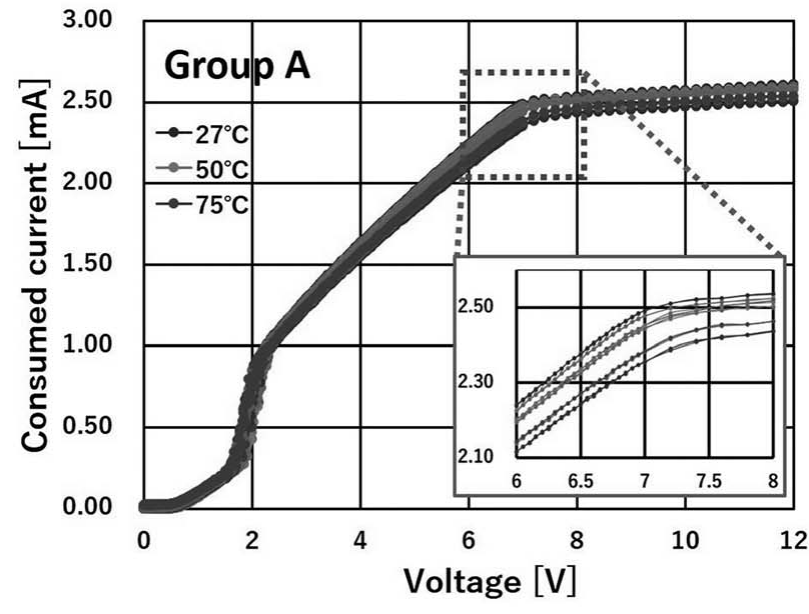

(b)

Fig. 2. The total consumption current of OPAs as a function of the supply voltage measured at room temperature. Groups A, B, and C indicate different product manufacturing lot numbers (a). The ambient temperature dependence for a typical sample device from Group A (b). 


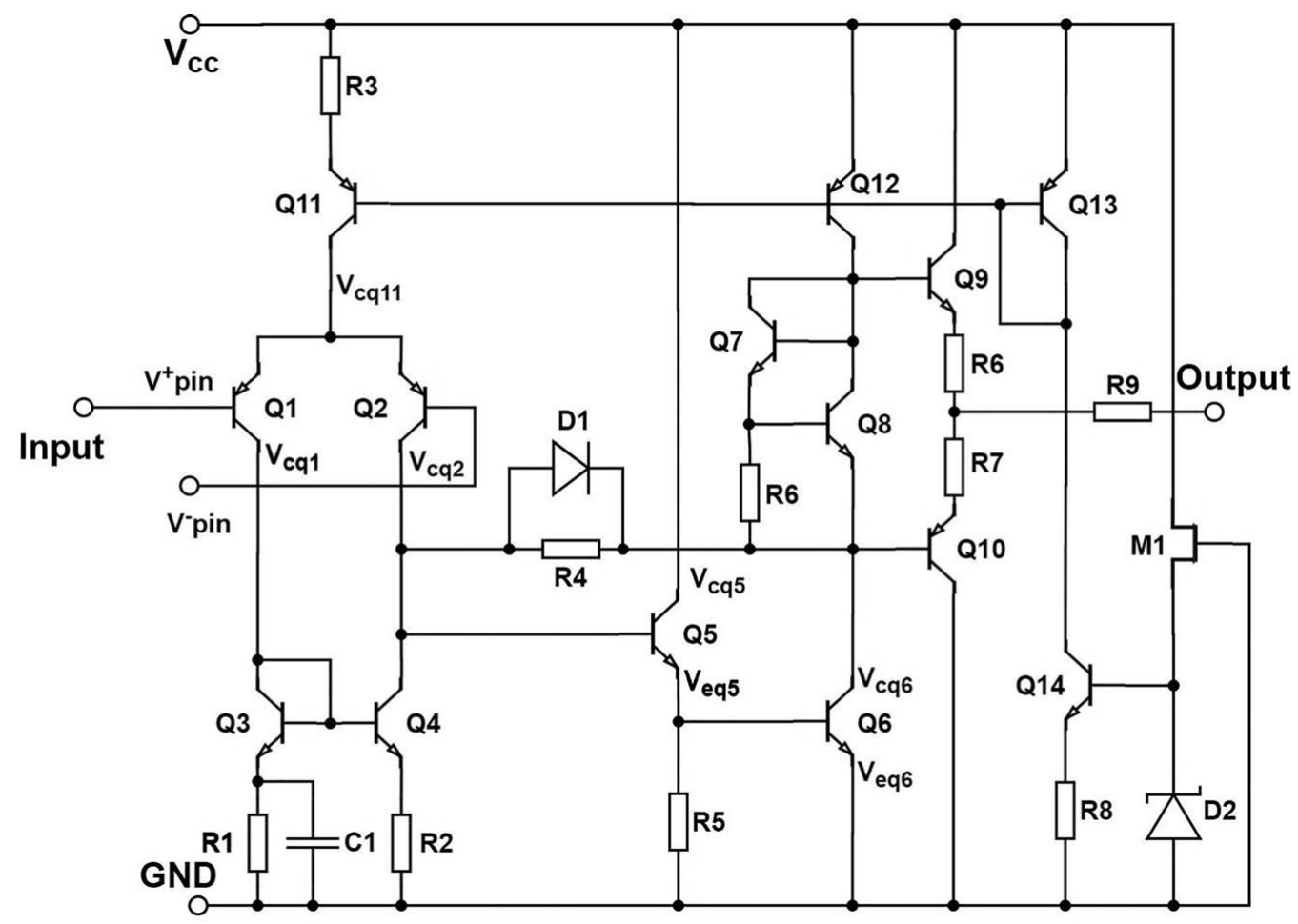

Fig. 3. A simplified circuit schematic of the BJT OPA NJM4558 [22].

The power supply voltage for the gain in OPA2 is provided via terminal VCC2 by an NJM2392 DC/DC converter. The output voltage is monitored by variable resistor VR4 and R11, and the voltage is fed back to change the duty ratio of the pulse-width modulation (PWM) signal $(100 \mathrm{kHz})$ to produce the desired power supply voltage $\mathrm{VCC} 2$, which ranges from $10 \mathrm{~V}$ down to $5 \mathrm{~V}$.

Fig. 5 shows a photograph of the new distortion pedal. The "Gain" knob (D1) controls the variable resistor VR1 to tune the gain of OPA2, as shown in (a). The "Variable DC" knob (D2) controls the variable resistor VR4 to tune the starved voltage VCC2 for OPA2, as shown in (b). In this research the tone control knob, which controls variable resistor VR2, was fixed in the zero position such that the corner frequency of the RC filter was set to $100 \mathrm{kHz}$.

\section{MEASUREMENT SYSTEM}

The nonlinear transfer characteristics of the distortion pedal were observed by inputting a one-tone signal and two-tone signal generated by a Keysight 35500B waveform generator into the distortion pedal. The frequency accuracy of this waveform generator is $1 \mu \mathrm{Hz}$ in the wide band from $1 \mu \mathrm{Hz}$ to $20 \mathrm{MHz}$, and the signal amplitude is controlled by a 16-bit digital-to-analog converter. This accuracy is sufficient to generate individual frequencies of equal temperament and the harmonic spectrum of the IMD of the distortion pedal output.
The nonlinear transfer characteristic was generated by varying both the "Gain" knob (VR1), named Distortion 1 (D1), and the "Variable DC" knob (VR4), named Distortion 2 (D2). The output power was equalized to yield a constant perceived loudness for human ears by using the "Level" knob (VR3) in accordance with the loudness, K-weighted, relative to the full scale (LKFS) standard for both the one-tone harmonic distortion test and two-tone IMD test. To perform the LKFS correction [23], the output signal of the distortion pedal was monitored with the Orban Loudness Meter V2.9.7 PC software application [24] via a USB-connected RME Babyface Pro AD converter. An Agilent DSO-X2024A digital storage oscilloscope was used to sample the output waveforms in all experiments.

First, a sine sweep analysis and an amplitude sweep analysis were performed using the continuous wavelet transform (CWT) in MATLAB Wavelet Toolbox version R2019a to determine the trend of the harmonic intensity of the output waveforms. The fast Fourier transform (FFT) function of the digital storage oscilloscope was then used for detailed analysis of the spectra. In this digital oscilloscope, the number of sampling points in a data stream is fixed to 50,000 . Therefore the frequency accuracy of the spectrum is defined by the combination of the recording time and frequency range. Here we performed 2-second recordings for FFT. This result corresponds to 2.5 times oversampling for the Nyquist frequency of $5 \mathrm{kHz}$ to capture the harmonics of the major frequency band of an electric guitar, 
(a)
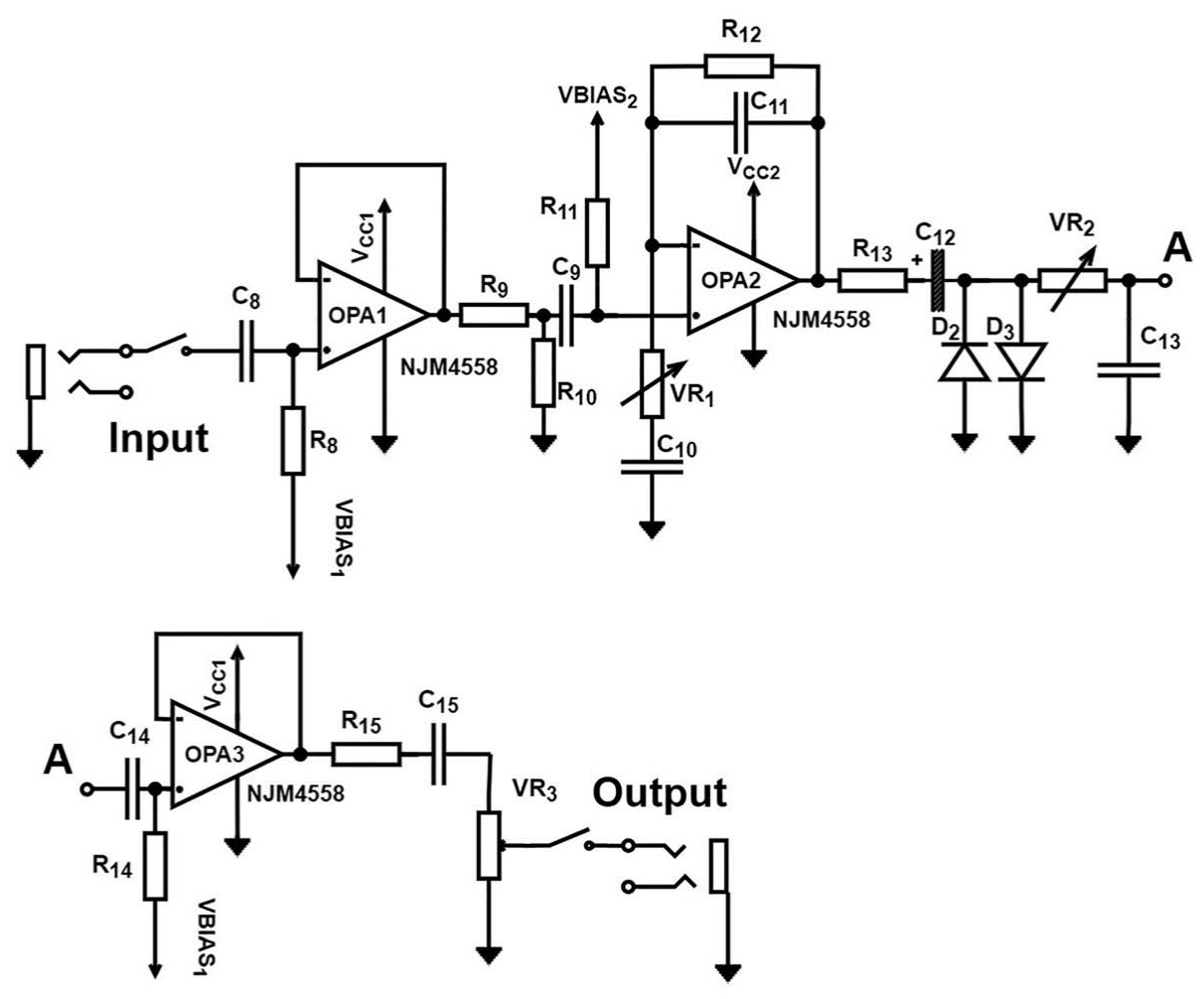

(b)

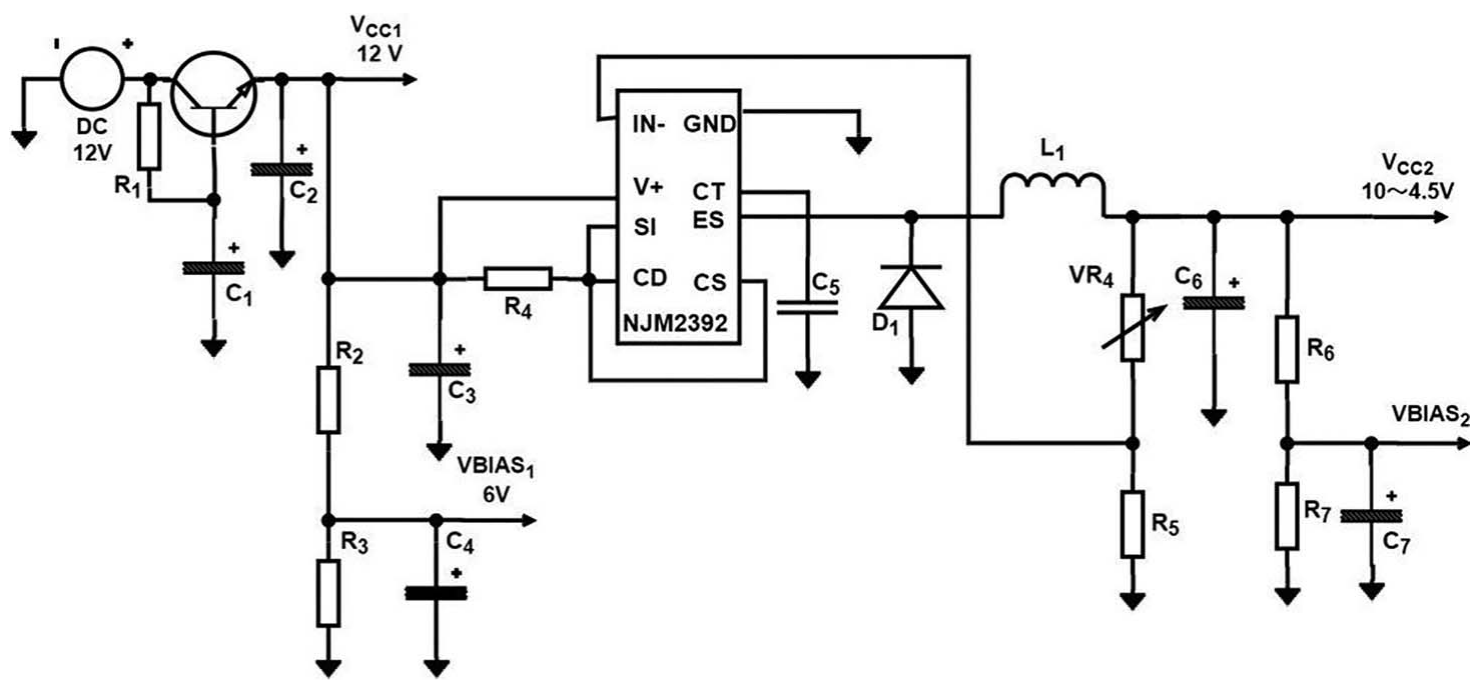

Fig. 4. Schematics of the developed distortion pedal: the signal processing circuit (a) and power supply management circuit (b).

which covers the maximum frequency of the keyboard pitch $\mathrm{C} 8=4.186 \mathrm{kHz}$.

\section{FUNDAMENTAL RESPONSE OF THE STARVATION-CONTROLLED DISTORTION PEDAL}

\subsection{Sine Sweep Spectrograms}

To observe the frequency range of the harmonics produced by the distortion pedal, sine sweeps were performed from $20 \mathrm{~Hz}$ to $2 \mathrm{kHz}$, covering the fundamental frequency range of an electric guitar ( $82 \mathrm{~Hz}$ to $1.175 \mathrm{kHz})$ with standard tuning. Fig. 6(a), (b), (c), (d), and (e) show the sine sweep spectrograms for distortion knob scale settings of 'D1 = 0, D2 = 0,' 'D1 = 0, D2 = 100, ' $\mathrm{D} 1=50, \mathrm{D} 2=$ $50,{ }^{\prime} \mathrm{D} 1=100, \mathrm{D} 2=0$,' and 'D $1=100, \mathrm{D} 2=100$,' respectively. In the shaded region outside the dashed black line in each panel, the information in the spectrogram should be treated as suspect due to the potential for edge effects [25]. The LKFS correction was applied for each distortion knob 
condition based on the average amplitude during the sine sweep, while the input signal amplitude was fixed to 400 $\mathrm{mVp}-\mathrm{p}$.

In the case of the no-effect condition ' $\mathrm{D} 1=0, \mathrm{D} 2=0$ ' panel (a), even-order ( $2^{\text {nd }}$ and $\left.4^{\text {th }}\right)$ harmonics are observed in addition to odd-order $\left(3^{\text {rd }}\right.$ and $\left.5^{\text {th }}\right)$ harmonics. With a voltage starvation of D2 $=100$, as seen in panel (b), the strength of the even-order harmonics becomes greater than that of the odd-order harmonics. A similar graph is observed when 'D1 $=50, \mathrm{D} 2=50$,' shown in panel (c). This panel indicates that the asymmetry of the OPA increases with the gain D1 as well as the supply voltage starvation D2. When the gain of the OPA is increased to D1 $=100$ with D2 $=0$, as seen in panel (d), the strength of the even-order harmonics increases, while when the supply voltage starvation is also increased to D2 $=100$, the odd-order harmonics decrease and almost vanish, as seen in panel (e).

\subsection{Amplitude Sweep Spectrograms}

Amplitude sweeps were performed from $50 \mathrm{mVp}$-p to $800 \mathrm{mVp}$-p for a sinusoidal wave with a concert pitch of $\mathrm{A}=440 \mathrm{~Hz}$. The maximum voltage of $800 \mathrm{mVp}$-p was determined from measurements using open $\mathrm{E}$ chords of a bridge humbucker under hard picking.

Fig. 7(a), (b), (c), (d), and (e) show the amplitude sweep spectrograms for distortion knob scale settings of ' $\mathrm{D} 1=0$, $\mathrm{D} 2=0$, 'D1 =0, D2 = 100,' 'D1 = 50, D2 = 50,' 'D1 $=100, \mathrm{D} 2=0$,' 'D1 = 100, D2 = 100,' and 'D1 = 100, $\mathrm{D} 2=100$,' respectively. The LKFS correction was applied over the entire sweep range based on an amplitude of 400 $\mathrm{mV}$ - $\mathrm{p}$ for each distortion knob condition. A strong input amplitude dependence is observed in the panels showing the 'D1 = 0, D2 = 0,' 'D1 =0, D2 = 100,' and 'D1 = 50, $\mathrm{D} 2=50$ ' cases. Note that in the ' $\mathrm{D} 1=0, \mathrm{D} 2=0$ ' panel (a), the $2^{\text {nd }}$-order harmonics increase with an increasing input amplitude, as do the $3^{\text {rd }}$-order harmonics, and the $4^{\text {th }}$-order harmonics are observed at amplitudes larger than approximately $250 \mathrm{mVp}$-p.

For the maximum voltage starvation case of ' $\mathrm{D} 1=0$, D2 $=100$ ' shown in panel (b), the $3^{\text {rd }}$-order harmonics are decreased at amplitudes above $300 \mathrm{mVp}$-p, while the evenorder harmonics are increased. A similar trend can be seen in panel (c), where the output is increased by the OPA gain, since the D1 knob is set to 50 in this case. Here the D2 knob is also set to 50, and although the degree of power supply starvation is less than that in panel (a), the headroom of the internal $3^{\text {rd }}$ high-gain stage is further reduced in response to the input amplitude of the $1^{\text {st }}$ differential stage of the OPA.

On the other hand, as shown in panels (d) and (e), the two cases with D1 $=100$ show almost no amplitude dependence. This result indicates that regardless of the input amplitude, a very high gain (D1 = 100) causes internal hard clipping by the supply voltage rails in the $4^{\text {th }}$ and $3^{\text {rd }}$ stages of the gain OPA. The strength of the even-order $\left(2^{\text {nd }}\right.$ and $4^{\text {th }}$ ) harmonics is very high compared to that of the odd-order ( $3^{\text {rd }}$ and $5^{\text {th }}$ ) harmonics; for 'D1 $=100$, D2 $=$ 100 ,' the even-order harmonics are dominant, whereas the strength of the odd-order harmonics is very weak.

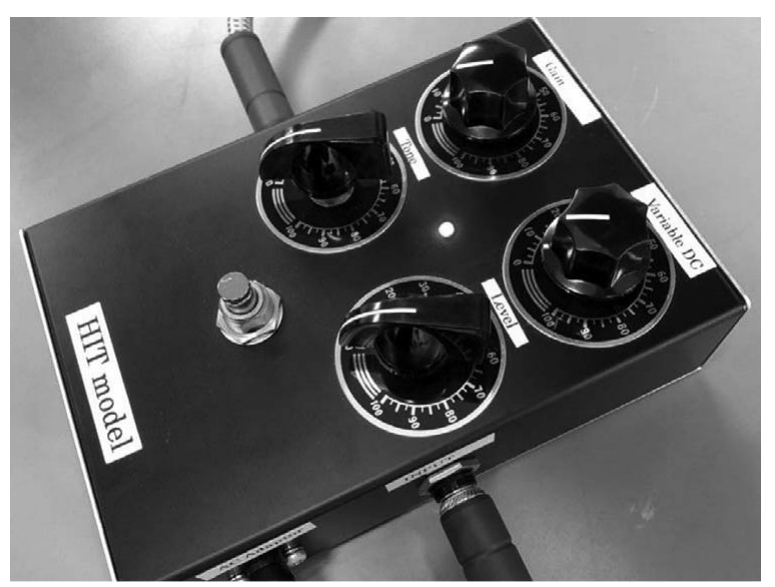

Fig. 5. The newly fabricated distortion pedal has 4 knobs: "Gain," "Tone," "Level," and a "Variable DC" knob that controls the feedback resistance VR4. The variation in distortion is controlled by a combination of the "Gain" knob D1 and "Variable DC" knob D2.

\section{TRANSFER FUNCTION OBTAINED FOR ONE-TONE HARMONIC DISTORTION}

\subsection{Transfer Function Measurement}

Fig. 8(a) shows the transfer characteristics with the distortion knob scale setting ' $\mathrm{D} 1=0, \mathrm{D} 2=0$ ' for the one-tone concert pitch $440 \mathrm{~Hz}$. Strong hysteresis is observed, which suggests phase differences between upward and downward transfer characteristics. The hysteresis depends on the signal frequency and is reduced to almost null by decreasing the frequency to $297 \mathrm{~Hz}$, thus compensating for the phase differences, as shown in Fig. 8(b). Therefore this curve is considered to represent the transfer function for the specific distortion condition of 'D $1=0, \mathrm{D} 2=0$.' Thus, based on the superposition principle for linear systems, the transfer function can be represented by a polynomial approximation that is thought to make real-time processing easier compared to the numerical methods introduced in SEC. 0.

\subsection{Polynomial Approximation of the Transfer Function}

To express the nonlinear transfer function with a memory effect such as IM3 in a radio frequency (RF) amplifier, the Volterra series is considered to be a superior approximation [26]. However, as mentioned in the previous section, the phase compensated transfer function can be approximated by an adequate order of polynomial function. Eq. (1) shows a third order polynomial as an example:

$$
\mathrm{V}_{\text {out }}=\mathrm{K}_{1} \mathrm{~V}_{\text {in }}+\mathrm{K}_{2}\left(\mathrm{~V}_{\text {in }}\right)^{2}+\mathrm{K}_{3}\left(\mathrm{~V}_{\text {in }}\right)^{3}
$$

Eq. (2) is given for a one-tone signal with an angular frequency of $\omega_{1}$.

$$
\mathrm{V}_{\text {in }}=A \cos \omega_{1} \mathrm{t}
$$




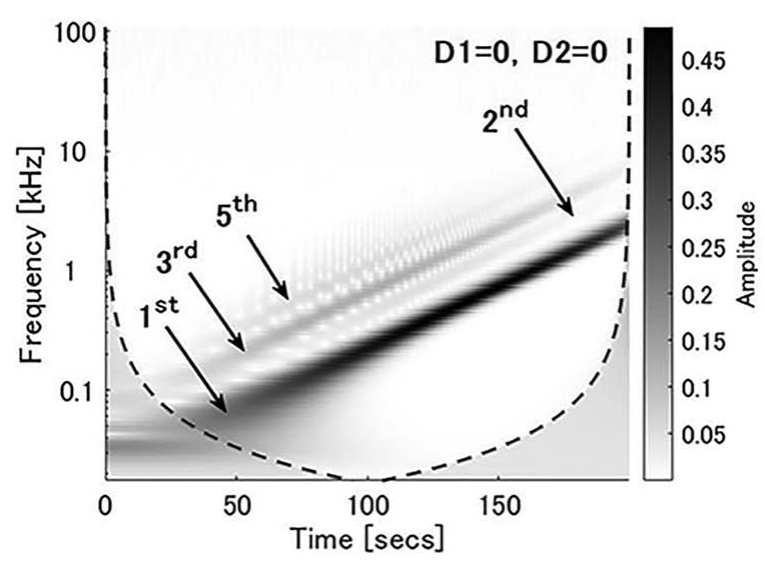

(a)

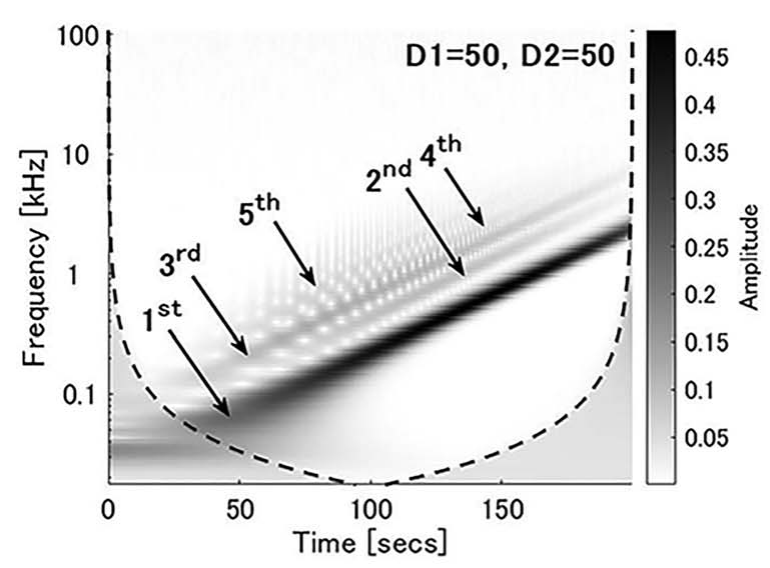

(c)

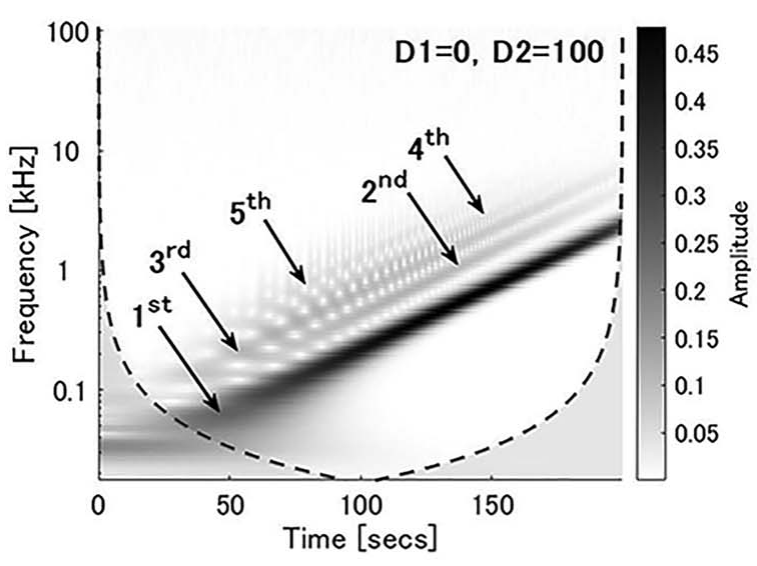

(b)

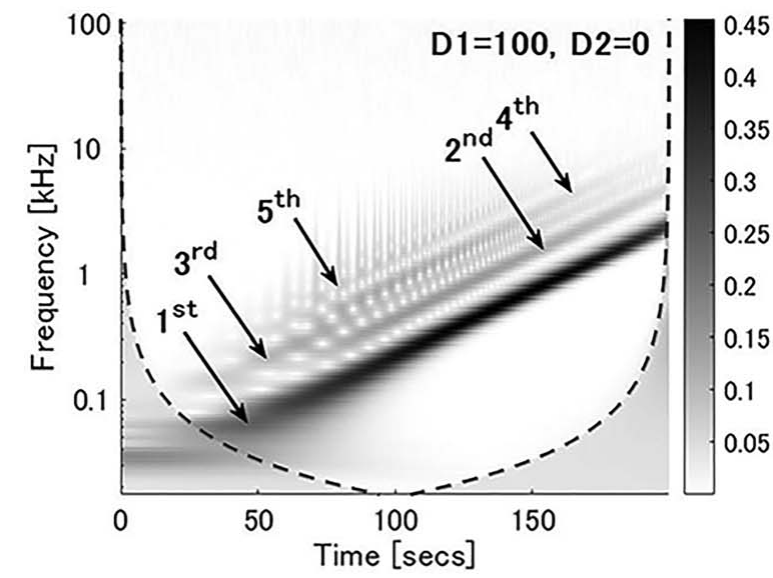

(d)

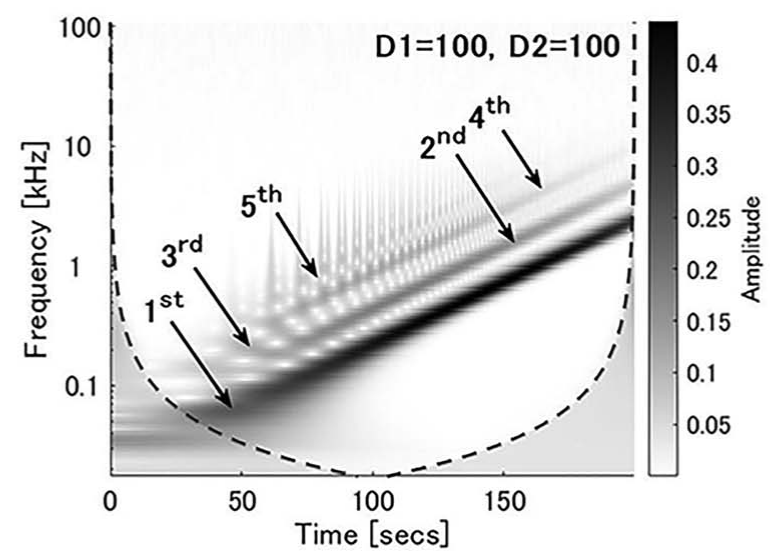

(e)

Fig. 6. The sine sweep spectrogram for distortion knob scale settings of ' $\mathrm{D} 1=0, \mathrm{D} 2=0$,' 'D1 = 0, D2 = 100,' 'D1 = 50, D2 = 50,' 'D1 = 100, D2 = 0,' and 'D1 = 100, D2 = 100' in (a), (b), (c), (d), and (e), respectively. Outside the dashed black line in the shaded region, information in the spectrogram should be treated as suspect due to the potential for edge effects [25]. 


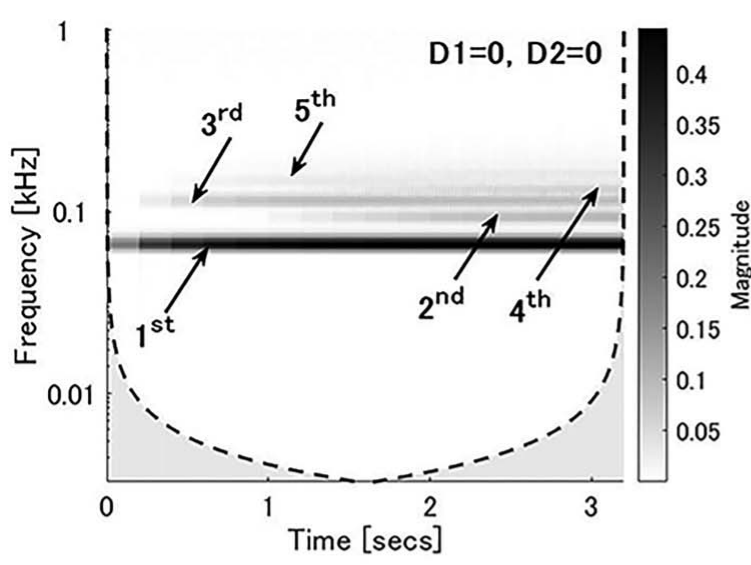

(a)

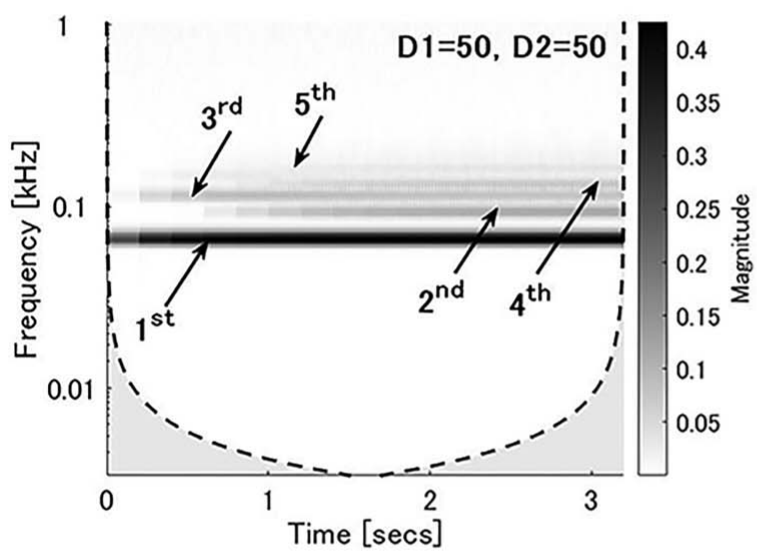

(c)

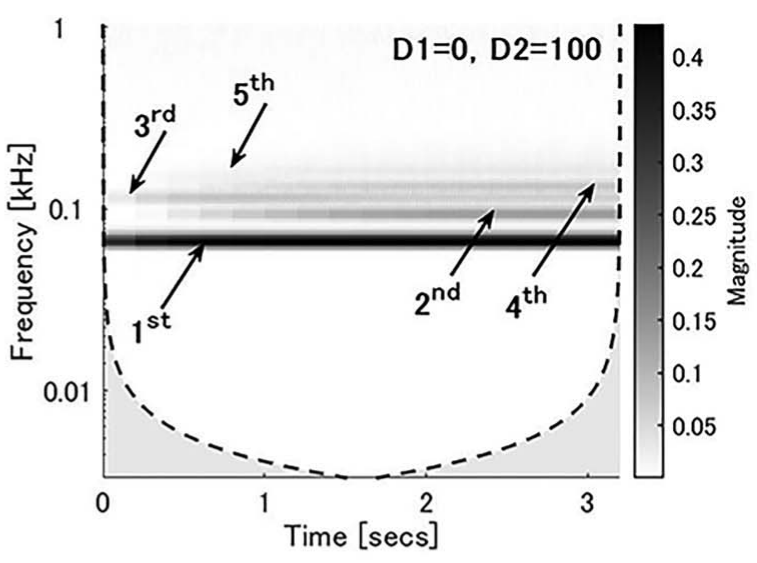

(b)

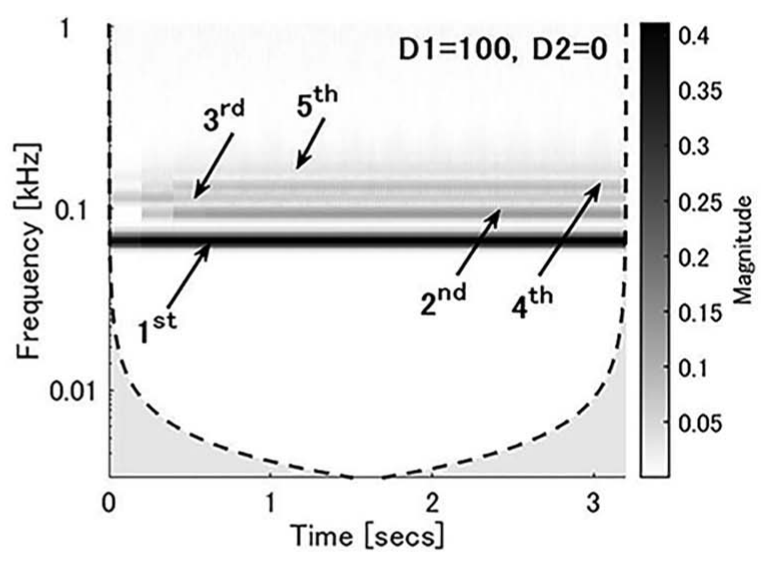

(d)

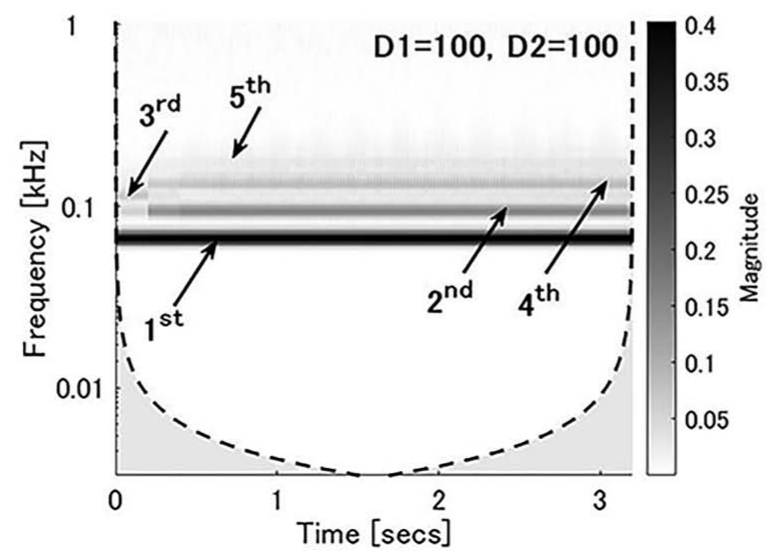

(e)

Fig. 7. The amplitude sweep spectrogram for distortion knob scale settings of ' $\mathrm{D} 1=0, \mathrm{D} 2=0$,' 'D1 $=0, \mathrm{D} 2=100$, ' $\mathrm{D} 1=50, \mathrm{D} 2=$ 50 ,' 'D1 = 100, D2 = 0,' and 'D1 = 100, D2 = 100' in (a), (b), (c), (d), and (e), respectively. In the shaded region, information in the spectrogram should be treated as suspect because of the potential for edge effects [25]. 


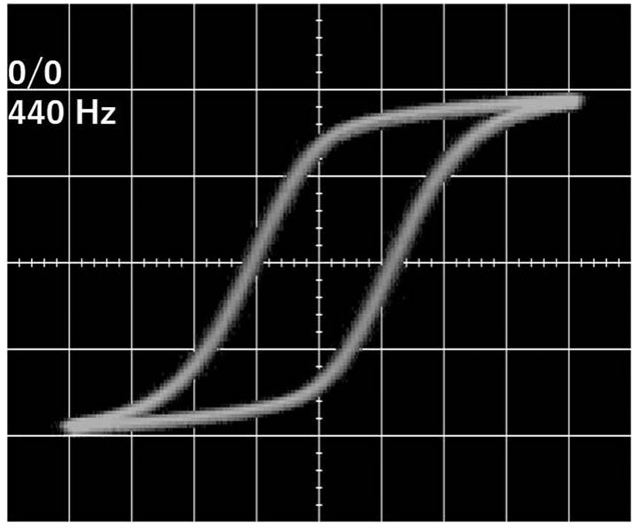

(a)

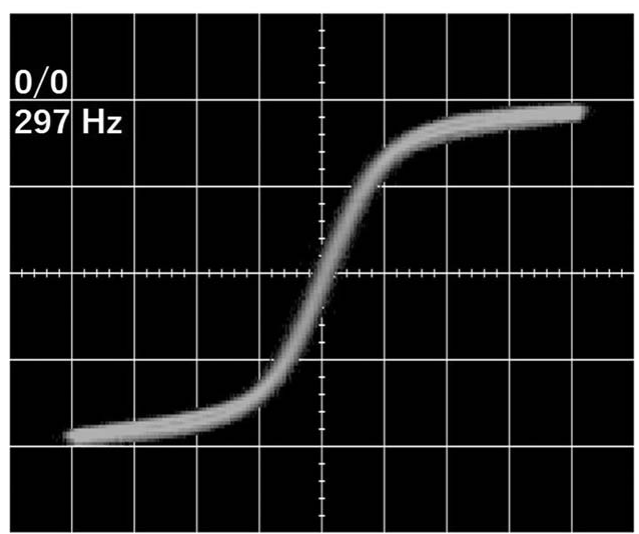

(b)

Fig. 8. Transfer characteristics of the default settings of distortion knob scales 'D1 $=0, \mathrm{D} 2=0$ ' for a one-tone concert pitch $\mathrm{A}=$ $440 \mathrm{~Hz}$. Phase modulated hysteresis is observed in (a), and the phase compensated for a one-tone signal of $297 \mathrm{~Hz}$ is shown in (b). The horizontal and vertical axes are the input amplitude (50 $\mathrm{mV} /$ div) and output amplitude (200 mV/div), respectively.

By substituting Eq. (2) into Eq. (1), Eq. (3) is obtained.

$$
\begin{aligned}
\mathrm{V}_{\text {out }}= & \mathrm{K}_{2} \mathrm{~A}^{2} / 2+\left\{\left(\mathrm{K}_{1} \mathrm{~A}\right)+\left(3 \mathrm{~K}_{3} \mathrm{~A}^{3} / 4\right)\right\} \cos \omega_{1} \mathrm{t} \\
& +\left(\mathrm{K}_{2} \mathrm{~A}^{2} / 2\right) \cos 2 \omega_{1} \mathrm{t}+\left(\mathrm{K}_{3} \mathrm{~A}^{3} / 4\right) \cos 3 \omega_{1} \mathrm{t}
\end{aligned}
$$

Higher-order terms can also be obtained similarly, and the higher-order coefficients significantly increase in complexity with an increasing power factor. When applying the least-squares method to both curves in Fig. 8(a) and (b), we choose a $9^{\text {th }}$-order polynomial. Thus polynomial approximation curves for the setting ' $\mathrm{D} 1=0, \mathrm{D} 2=0$ ' are obtained for the $440 \mathrm{~Hz}$ hysteretic curve and the $297 \mathrm{~Hz}$ phase-compensated curve, as shown in Fig. 9.

\subsection{Measurement and Simulation}

\subsubsection{Default Distortion Conditions}

Spectra obtained through simulation with the $440 \mathrm{~Hz}$ and $297 \mathrm{~Hz}$ polynomial approximation functions obtained as described above are compared with the measured spectra in Fig. 10. (Note: the $440 \mathrm{~Hz}$ one-tone input was used in both cases of measurement and the two transfer functions were expressed as polynomial approximations.) Here

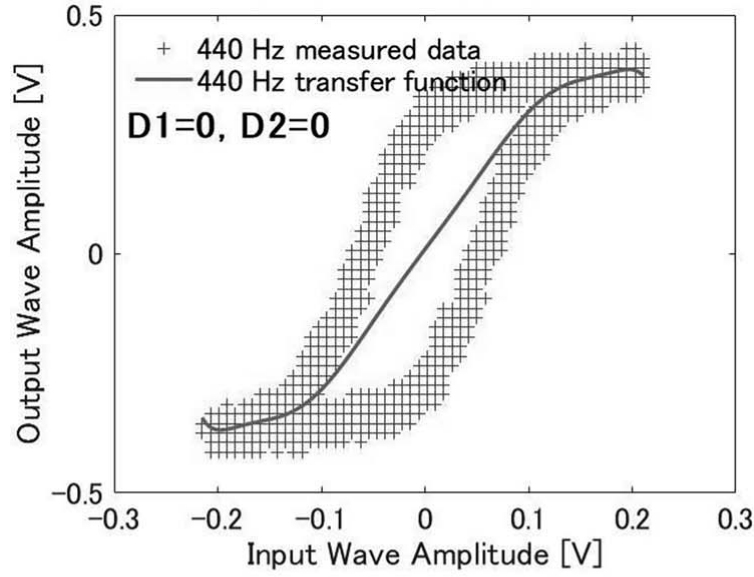

(a)

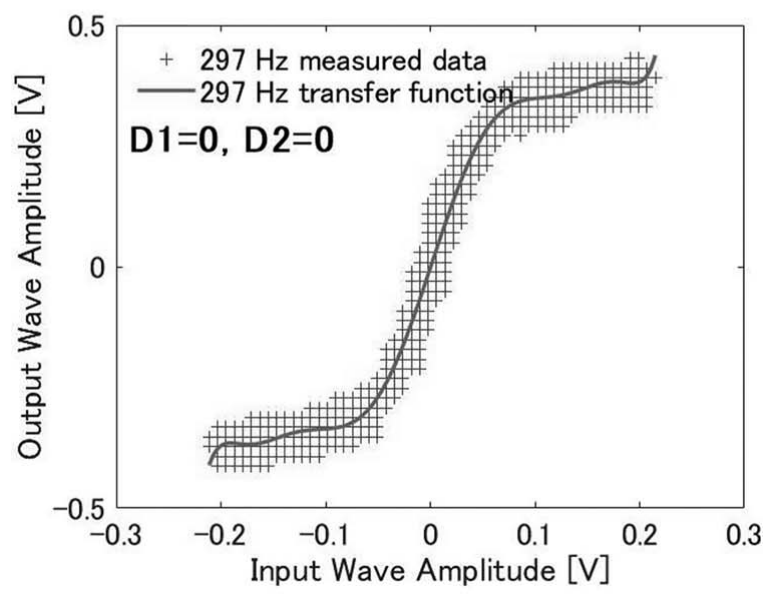

(b)

Fig. 9. Transfer characteristics for a one-tone concert pitch $\mathrm{A}=440 \mathrm{~Hz}$ (a) and for a phase-compensated one-tone signal by decreasing the frequency to $297 \mathrm{~Hz}$ (b) in the case of distortion knobs ' $\mathrm{D} 1=0, \mathrm{D} 2=0$.' The + symbol indicates measurement data and the solid line is a curve simulated by a $9^{\text {th }}$-order polynomial approximation.

the simulated spectra were obtained using the following process in MATLAB version R2019a:

1. An input signal tone stream with 50,000 points $(2 \mathrm{~s})$ was digitized to match the measurement conditions.

2. This signal stream was input into the transfer function to obtain the corresponding output signal.

3. The FFT was performed on the output signal stream. Thus the frequency resolution of the spectrum was truncated to $0.5 \mathrm{~Hz}$; this is smaller than the human "just-noticeable frequency difference," which has a constant value of approximately $1.3 \mathrm{~Hz}$ below a test signal tone frequency $f$ of $500 \mathrm{~Hz}$ and is equal to 1 $+0.007 f \mathrm{~Hz}$ above $1 \mathrm{kHz}$ [27].

Fig. 11 shows the correlations between the amplitudes of the measured and simulated harmonics down to $-50 \mathrm{dBV}$. Interestingly the $297 \mathrm{~Hz}$ polynomial, fitted for the phasecompensated transfer function, reproduces the measured 


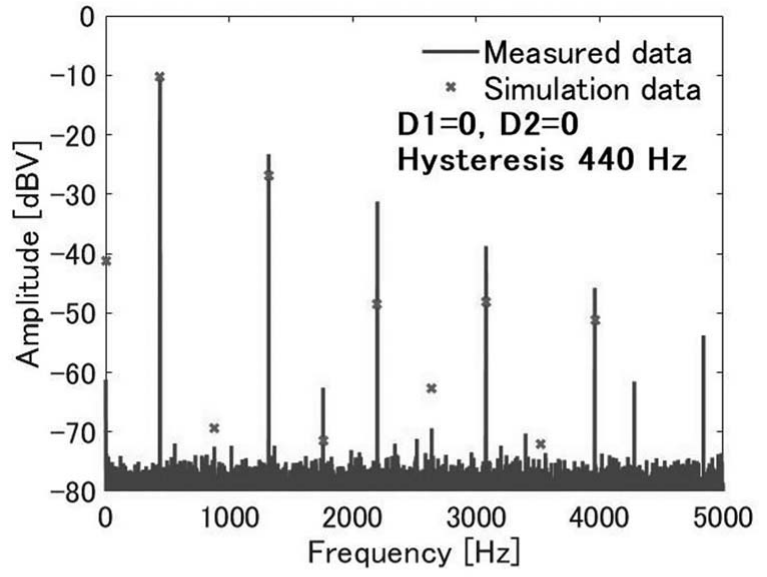

(a)

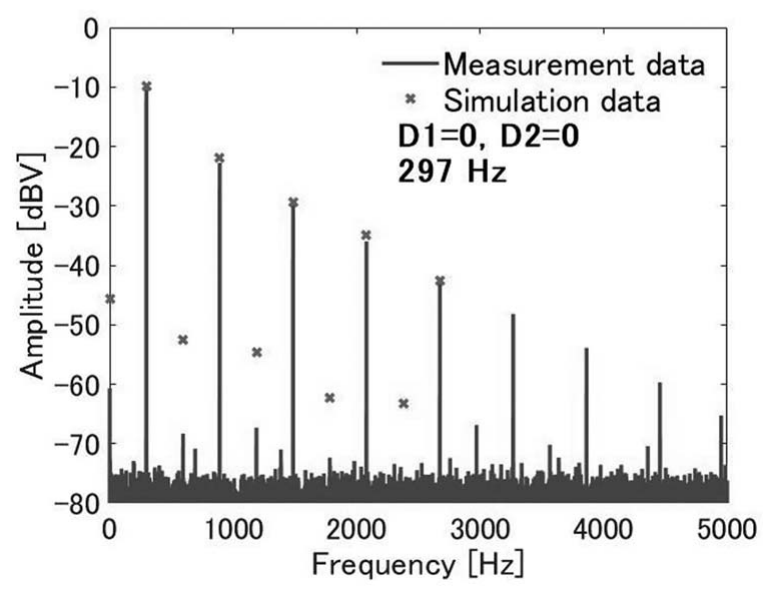

(b)

Fig. 10. Measured spectra of the distortion pedal output for an input signal of $440 \mathrm{~Hz}$ and simulated spectra using a polynomial approximation optimized by a hysteresis curve of $440 \mathrm{~Hz}$ (a) and phase-compensated curve of $297 \mathrm{~Hz}$ (b).

Table 1. Input signal frequency of the phase compensation for various knob value combinations of Distortion 1 and

Distortion2. In the case of 'D1 $=100, \mathrm{D} 2=100$,' the original frequency of $440 \mathrm{~Hz}$ yields no hysteresis.

\begin{tabular}{llll}
\hline D1 \D2 & 0 & 50 & 100 \\
\hline 0 & $297 \mathrm{~Hz}$ & $295 \mathrm{~Hz}$ & $276 \mathrm{~Hz}$ \\
50 & $350 \mathrm{~Hz}$ & $340 \mathrm{~Hz}$ & $296 \mathrm{~Hz}$ \\
100 & $550 \mathrm{~Hz}$ & $510 \mathrm{~Hz}$ & $440 \mathrm{~Hz}$ \\
\hline
\end{tabular}

harmonic amplitudes better than the $440 \mathrm{~Hz}$ polynomial. This result indicates that the amplitude modulation due to the nonlinearity of the static transfer function is significant for a one-tone signal. This phenomenon is also observed under the other distortion knob settings, as presented in the next subsection.

\subsubsection{Gain and Starvation Changes}

Fig. 12 illustrates similar results for 'D1 $=100, \mathrm{D} 2=$ 0 ' and 'D1 $=100, \mathrm{D} 2=100$.' The signal frequencies used for phase compensation are summarized in Table 1. The

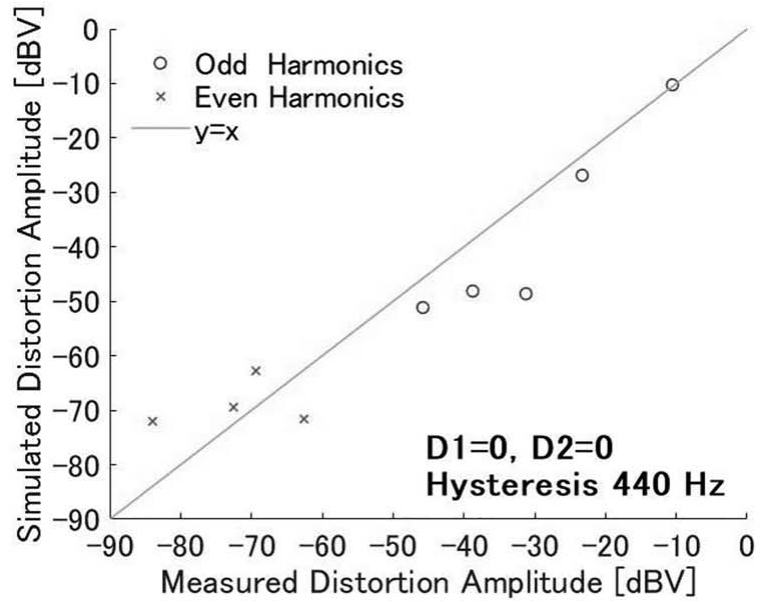

(a)

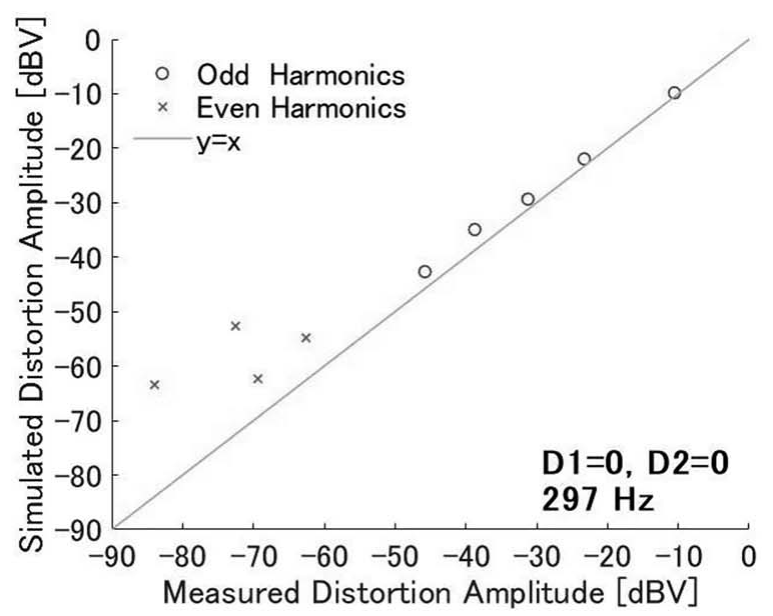

(b)

Fig. 11. Correlation of the harmonic amplitude strength between the measured spectra of the input signal, $440 \mathrm{~Hz}$, and the simulated spectra optimized by a hysteresis curve of $440 \mathrm{~Hz}$ (a) and a phasecompensated curve of $297 \mathrm{~Hz}$ (b). The open-circle and opensquare symbols indicate odd and even harmonics, respectively.

Distortion $1 \mathrm{knob}$, for gain, primarily controls the odd-order harmonics by enhancing the amplitude and distortion. The Distortion $2 \mathrm{knob}$, for voltage starvation, controls the evenorder harmonics. By comparing the differences between Fig. 12(a) and (b) and 12(c) and (d), it can be seen that the phase modulation is larger for odd-order nonlinearity than for even-order nonlinearity.

\section{TWO-TONE IMD}

For musical instruments, IMD between multiple tones is essential. In this section we analyze the dissonance and consonance IMD spectrum by considering the transfer function in a manner similar to that described in the previous section for two-tone signals of 12-tone equal temperament.

Here we must correctly define Dissonance and Consonance. The history of these definitions is described in detail in Tenney's text [28]. One of the earliest definitions is called the Pythagorean scale, which is based on a sequence 


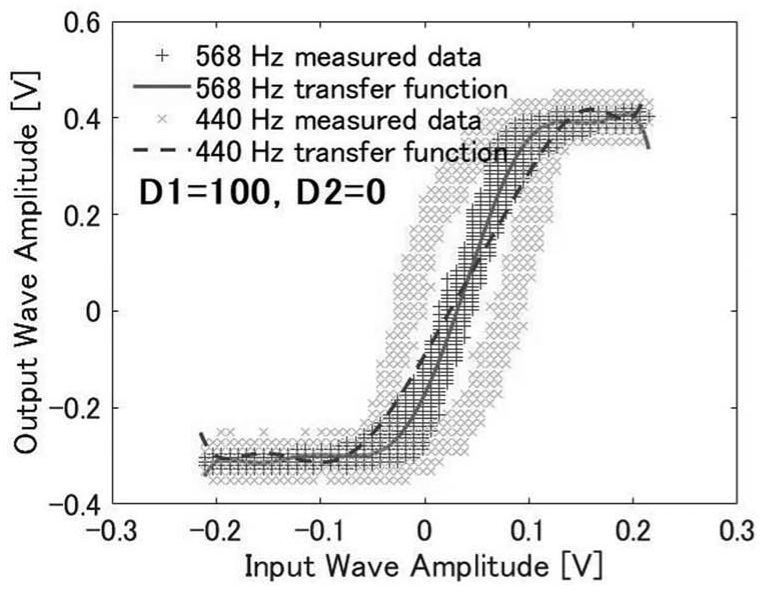

(a)

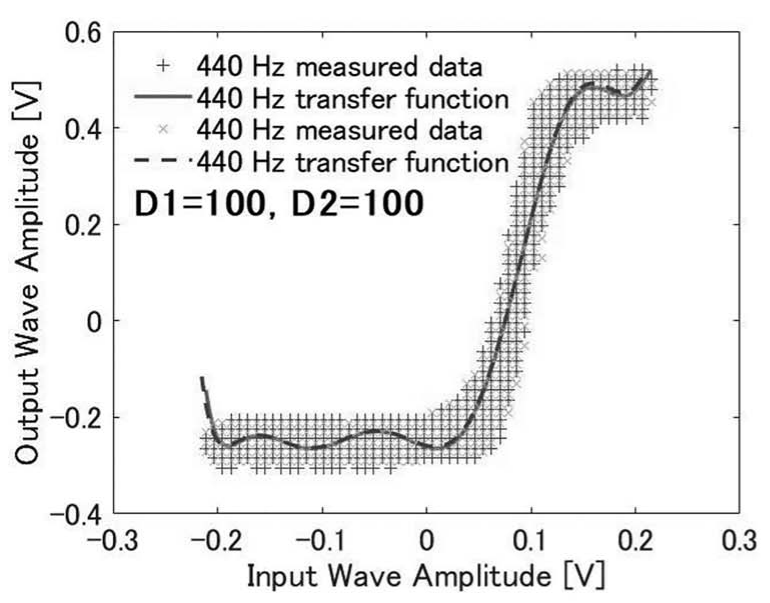

(c)

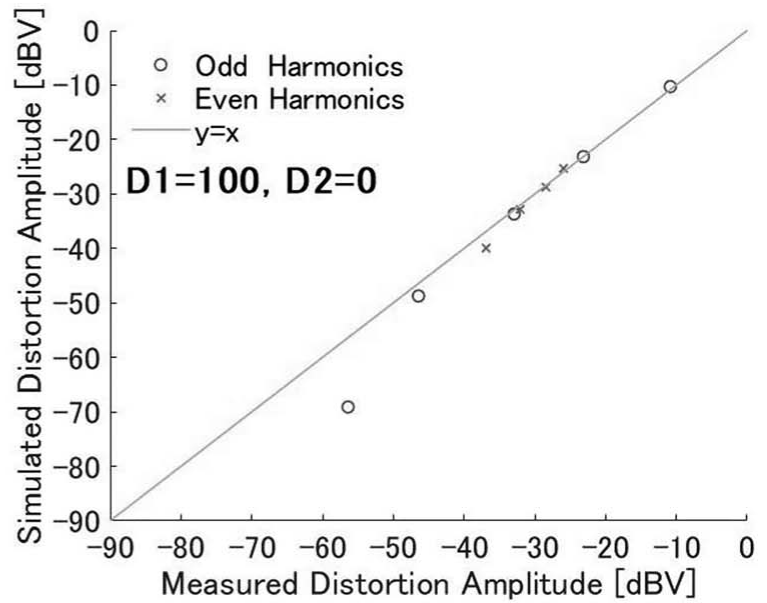

(b)

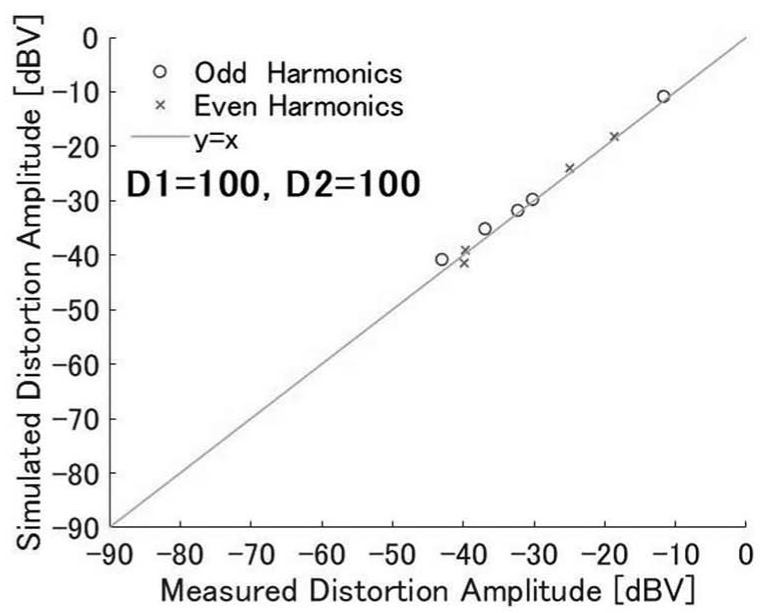

(d)

Fig. 12. Measured transfer characteristic for a one-tone concert pitch of $440 \mathrm{~Hz}$ and a phase-compensated polynomial curve in the case of distortion knob scales 'D1 $=100, \mathrm{D} 2=0$ ' (a) and 'D1 $=100, \mathrm{D} 2=100$ ' (c). Correlation of the harmonic strength between the measured spectra of the input signal of $440 \mathrm{~Hz}$ and the spectra simulated by a phase-compensated polynomial curve in the case of distortion knob scales 'D1 = 100, D2 = 0' (b) and 'D1 = 100, D2 = 100' (d).

of tuning in perfect fourths, fifths, and octaves. For example the interval ratio of "perfect fifth" is $3 / 2=1.5$. However, that of "12-tone equal temperament, perfect fifth" is $2^{7 / 12}$ $=1.4983$, which is not an integer ratio but is defined in music theory as consonance. Therefore, for the purpose of physically observing the IMD spectrum between two tones defined by "12-tone equal temperament," the following "physical rule" was adopted as definitions of dissonance and consonance [29] in page 194 of this article:

"When two musical tones are sounded at the same time, their united sound is generally disturbed by the beats of the upper partials, so that a greater or less part of the whole mass of sound is broken up into pulses of tone, and the joint effect is rough. This relation is called dissonance."

But "There are certain determinate ratios between pitch numbers, for which this rule suffers an exception, and either no beats at all are formed, or at least only such as have so little intensity that they produce no unpleasant disturbance of the united sound. These exceptional cases are called consonance."

\subsection{IMD for Dissonance}

\subsubsection{IMD Peaks Identified by Polynomial Approximation}

The dissonance between $\mathrm{f}_{1}=369.99 \mathrm{~Hz}\left(\mathrm{~F}_{\# 4}\right)$ and $\mathrm{f}_{2}$ $=587.33 \mathrm{~Hz}\left(\mathrm{D}_{5}\right)$ with a $1: 1$ amplitude ratio was chosen for IMD measurements because the difference in the intermodulation $(\mathrm{IM})$ frequencies, $\mathrm{f}_{2}-\mathrm{f}_{1}(587.33-369.99=$ $217.34 \mathrm{~Hz}$ ), is subjectively easy to notice. Furthermore the frequencies of the major IMD peaks are located in the band below $1 \mathrm{kHz}$ as shown in Fig. 13, in which some key psychoacoustic parameters are constant values independent of the frequency, such as the critical bandwidth, critical band level, and just-noticeable sound change [27].

To obtain the polynomial coefficients we formulated a combination of two angular frequencies, $\omega_{1}$ and $\omega_{2}$, as shown in Eq. (4), and then substituted Eq. (4) into Eq. (1).

$$
\mathrm{V}_{\mathrm{in}}=A \cos \omega_{1} \mathrm{t}+\mathrm{B} \cos \omega_{2} \mathrm{t}
$$




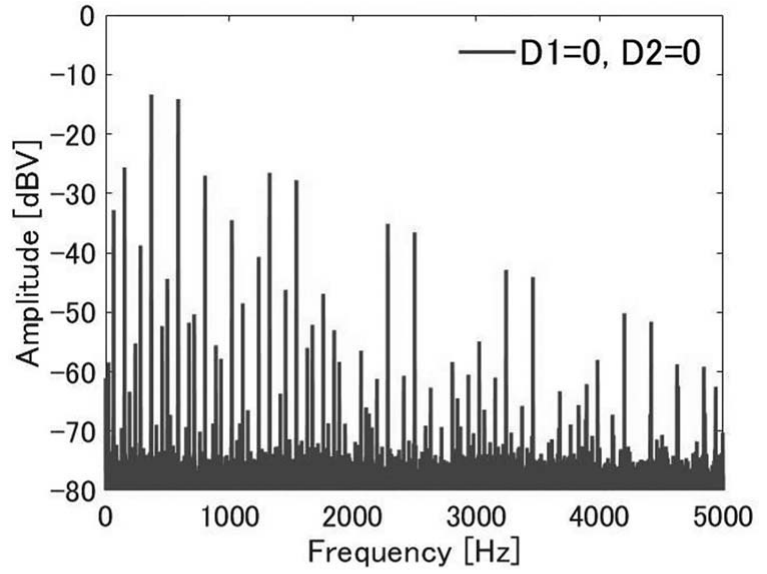

(a)

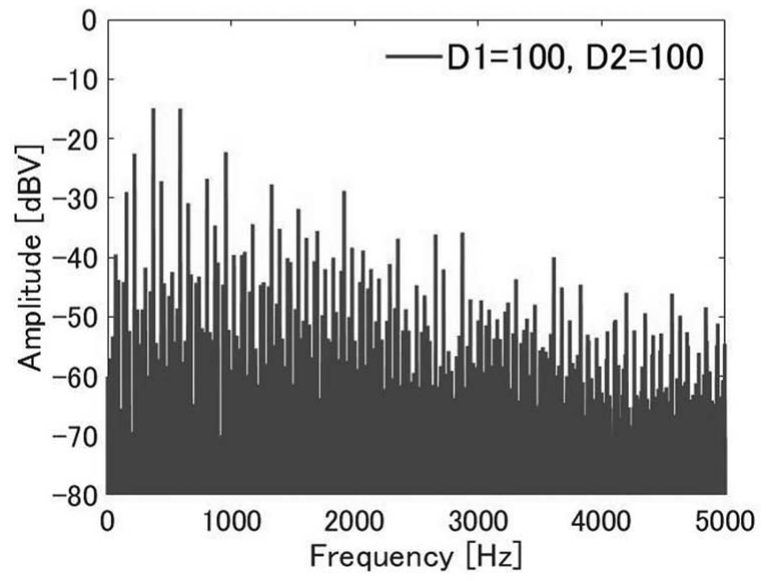

(b)

Fig. 13. Measured spectra of the distortion pedal output obtained by inputting signals $\mathrm{f}_{1}=369.99 \mathrm{~Hz}\left(\mathrm{~F}_{\# 4}\right)$ and $\mathrm{f}_{2}=587.33 \mathrm{~Hz}\left(\mathrm{D}_{5}\right)$ for distortion knob scales ' $\mathrm{D} 1=0, \mathrm{D} 2=0$ ' and 'D1 = 100, D2 $=100$ ' in (a) and (b), respectively.

As a simple example the derivation of the IMD to the third order is shown below (however, note that we applied the $9^{\text {th }}$-order approximation in this research):

$$
\begin{aligned}
\mathrm{V}_{\text {out }}= & \mathrm{V}_{0}+\mathrm{V}_{11} \cos \omega_{1} \mathrm{t}+\mathrm{V}_{12} \cos \omega_{2} \mathrm{t} \\
& +\mathrm{V}_{21} \cos 2 \omega_{1} \mathrm{t}+\mathrm{V}_{22} \cos 2 \omega_{2} \mathrm{t} \\
& +\mathrm{V}_{23}\left\{\cos \left(\omega_{1}+\omega_{2}\right)\right. \\
& \left.+\cos \left(\omega_{2}-\omega_{1}\right) \mathrm{t}\right\}+\mathrm{V}_{31} \cos 3 \omega_{1} \mathrm{t} \\
& +\mathrm{V}_{32} \cos 3 \omega_{2} \mathrm{t}+\mathrm{V}_{33}\left\{\cos \left(2 \omega_{1}+\omega_{2}\right) \mathrm{t}\right. \\
& \left.+\cos \left(2 \omega_{1}-\omega_{2}\right) \mathrm{t}\right\} \\
& +\mathrm{V}_{34}\left\{\cos \left(2 \omega_{2}+\omega_{1}\right) \mathrm{t}+\cos \left(2 \omega_{2}-\omega_{1}\right) \mathrm{t}\right\}
\end{aligned}
$$

$$
\begin{aligned}
& \text { where } \mathrm{V}_{0}=\mathrm{K}_{2}\left(\mathrm{~A}^{2}+\mathrm{B}^{2}\right) / 2, \\
& \mathrm{~V}_{11}=\left(\mathrm{K}_{1} \mathrm{~A}\right)+\left(3 \mathrm{~K}_{3} \mathrm{AB}^{2} / 2\right)+\left(3 \mathrm{~K}_{3} \mathrm{~A}^{3} / 4\right), \\
& \mathrm{V}_{12}=\left(\mathrm{K}_{1} \mathrm{~B}\right)+\left(3 \mathrm{~K}_{3} \mathrm{~A}^{2} \mathrm{~B} / 2\right)+\left(3 \mathrm{~K}_{3} \mathrm{~B}^{3} / 4\right), \\
& \mathrm{V}_{21}=\mathrm{K}_{2} \mathrm{~A}^{2} / 2, \\
& \mathrm{~V}_{22}=\mathrm{K}_{2} \mathrm{~B}^{2} / 2,
\end{aligned}
$$

Table 2. Some of the combinations of two-tone frequencies, summarized in order from small frequencies to $1,000 \mathrm{~Hz}$.

\begin{tabular}{ll}
\hline Frequency $[\mathrm{Hz}]$ & Formula \\
\hline 64.70 & $2 \mathrm{f}_{2}-3 \mathrm{f}_{1}$ \\
87.97 & $5 \mathrm{f}_{1}-3 \mathrm{f}_{1}$ \\
152.66 & $2 \mathrm{f}_{1}-\mathrm{f}_{2}$ \\
217.36 & $\mathrm{f}_{2}-\mathrm{f}_{1}$ \\
282.06 & $3 \mathrm{f}_{2}-4 \mathrm{f}_{1}$ \\
305.33 & $4 \mathrm{f}_{1}-2 \mathrm{f}_{2}$ \\
370.03 & $\mathrm{f}_{1}$ \\
434.65 & $2 \mathrm{f}_{2}-2 \mathrm{f}_{1}$ \\
457.92 & $6 \mathrm{f}_{1}-3 \mathrm{f}_{2}$ \\
499.34 & $4 \mathrm{f}_{2}-5 \mathrm{f}_{1}$ \\
522.61 & $3 \mathrm{f}_{1}-\mathrm{f}_{2}$ \\
587.31 & $\mathrm{f}_{2}$ \\
652.01 & $3 \mathrm{f}_{2}-3 \mathrm{f}_{1}$ \\
675.28 & $5 \mathrm{f}_{1}-2 \mathrm{f}_{2}$ \\
739.97 & $2 \mathrm{f}_{1}$ \\
804.67 & $2 \mathrm{f}_{2}-\mathrm{f}_{1}$ \\
869.37 & $4 \mathrm{f}_{2}-4 \mathrm{f}_{1}$ \\
892.64 & $4 \mathrm{f}_{1}-\mathrm{f}_{2}$ \\
957.34 & $\mathrm{f}_{1}+\mathrm{f}_{2}$ \\
\hline
\end{tabular}

$$
\begin{aligned}
\mathrm{V}_{23} & =\mathrm{K}_{2} \mathrm{AB}, \\
\mathrm{V}_{31} & =\mathrm{K}_{3} \mathrm{~A}^{3} / 4, \\
\mathrm{~V}_{32} & =\mathrm{K}_{3} \mathrm{~B}^{3} / 4, \\
\mathrm{~V}_{33} & =3 \mathrm{~K}_{3} \mathrm{~A}^{2} \mathrm{~B} / 4, \\
\mathrm{~V}_{34} & =3 \mathrm{~K}_{3} \mathrm{AB}^{2} / 4 .
\end{aligned}
$$

As shown in Eq. (5-1)-(5-11), a very large number of new tones appear. Table 2 summarizes some of the twotone frequency combinations from low frequencies to 1,000 $\mathrm{Hz}$, including the $9^{\text {th }}$-order frequencies $6 \mathrm{f}_{1}-3 \mathrm{f}_{2}$ and $4 \mathrm{f}_{2}-5 \mathrm{f}_{1}$ and the $8^{\text {th }}$-order frequencies $5 \mathrm{f}_{1}-3 \mathrm{f}_{2}$ and $4 \mathrm{f}_{2}-4 \mathrm{f}_{1}$. This table indicates that higher-order distortion peaks appear even in the lower-frequency band.

To automatically identify the tone peaks in the measured data, we developed a program using MATLAB. The algorithm steps are listed as follows:

- Frequency $=\mathrm{nf}_{1}+\mathrm{mf}_{2}(\mathrm{n}+\mathrm{m}$ is the order $)$

- $\mathrm{n}, \mathrm{m}(-9 \sim 9)$

- Frequency $>0$

- Extract the value of the closest Frequency from the oscilloscope CSV data

- Discrepancy is less than $0.1 \mathrm{~Hz}$

We identified $87 \mathrm{IM}$ peaks in the audio band up to $5 \mathrm{kHz}$ for the $2^{\text {nd }}$ to $9^{\text {th }}$ orders. This enabled us to compare the peak strengths of the measured peaks with the simulated peak strengths. As discussed for the case of a single tone, we again tested a polynomial approximation for the measured transfer characteristics of the IMD. As shown in Fig. 14 , no open space is apparent between the overlapped upward and downward hysteresis curves of the transfer characteristics for various IMD frequencies. The polynomial 


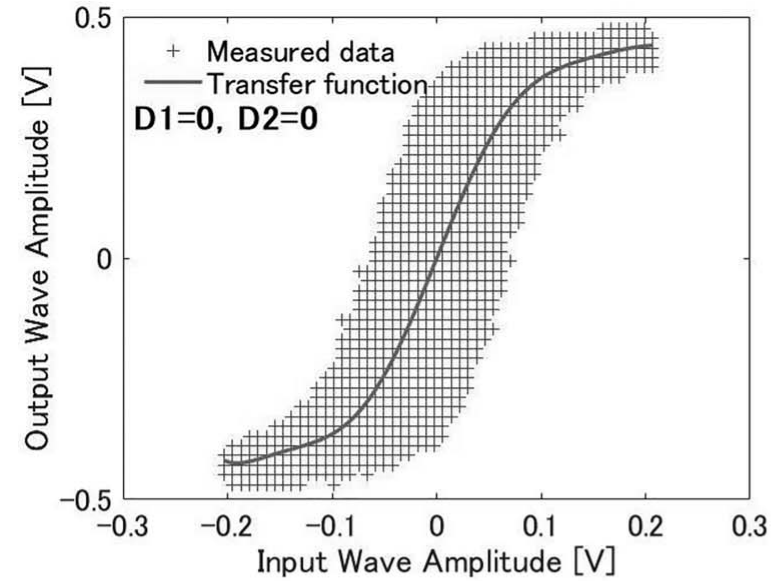

(a)

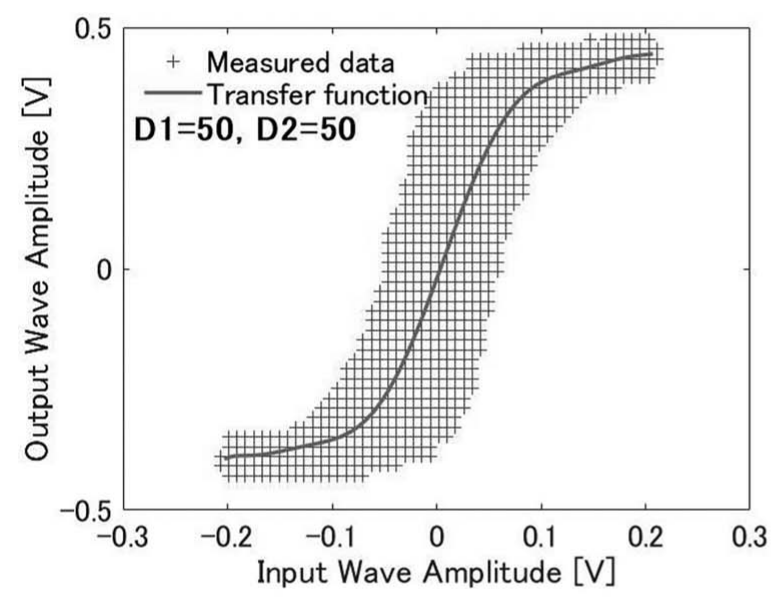

(c)

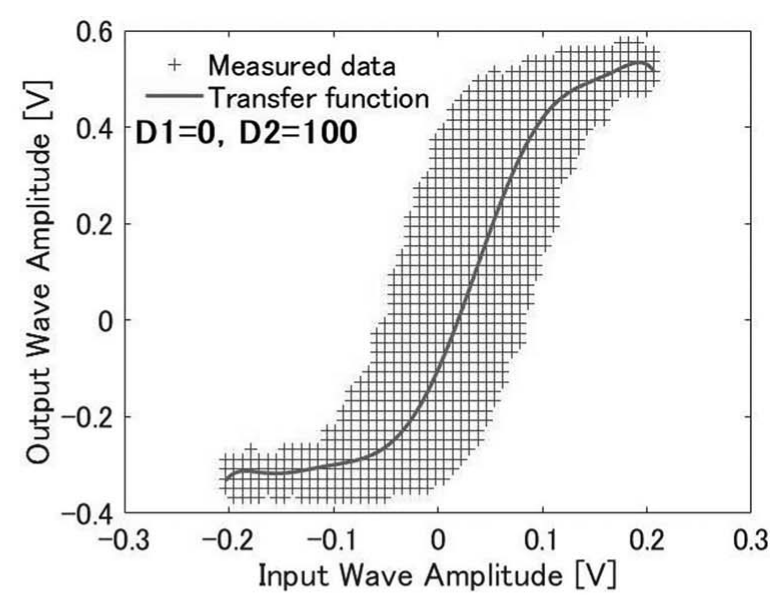

(b)

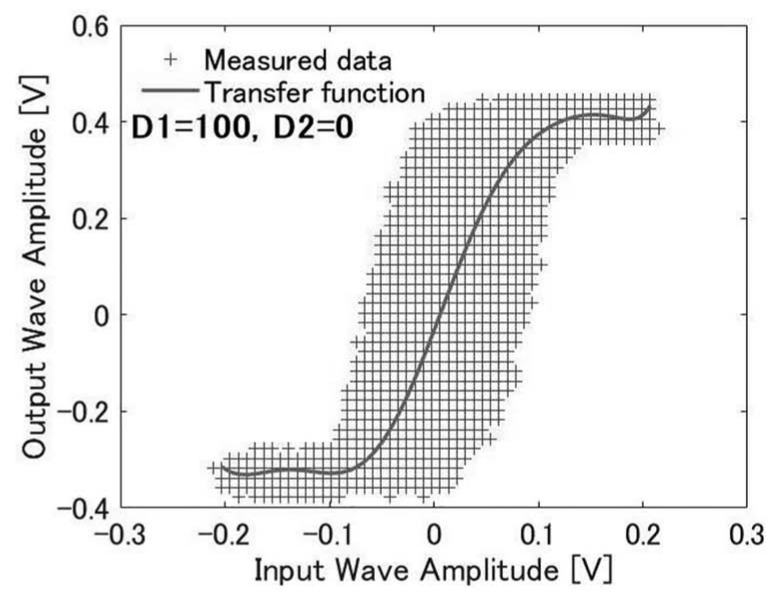

(d)

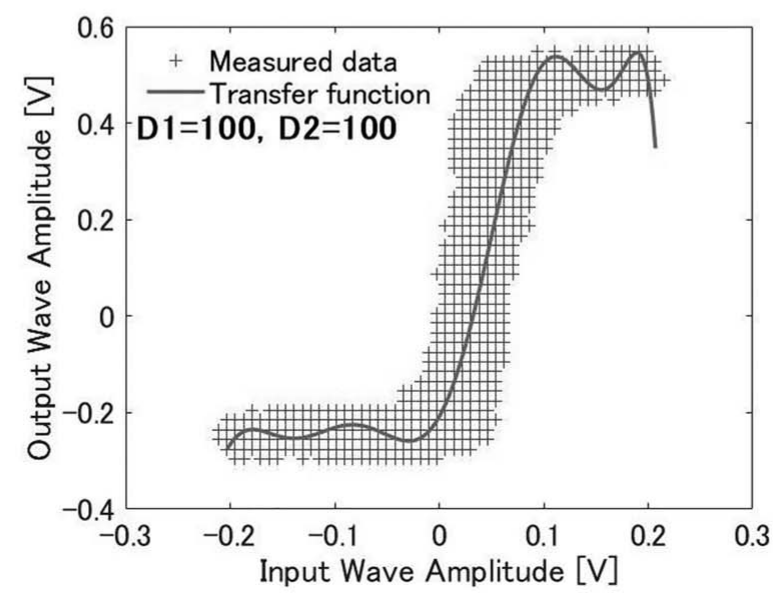

(e)

Fig. 14. Transfer characteristics of the IMD for distortion knob scale settings of 'D1 =0, D2 =0,' 'D1 = 0, D2 = 100, ' $\mathrm{D} 1=50, \mathrm{D} 2$ $=50$, ' $\mathrm{D} 1=100, \mathrm{D} 2=0$,' and 'D1 = 100, D2 = 100' in (a), (b), (c), (d), and (e), respectively. The polynomial curve fit is drawn in each graph. 
approximation curve was calculated up to the $9^{\text {th }}$ order via the least-squares method using all measured data points.

\subsubsection{Dependence of Dissonance IMD on Gain and Starvation: Measurement and Simulation}

The measured and simulated spectra of dissonant signals with distortion knob scale settings of ' $\mathrm{D} 1=0, \mathrm{D} 2=0$,' 'D1 = 0, D2 = 100,' 'D1 = 50, D2 = 50,' 'D1 = 100, $\mathrm{D} 2=0$,' and ' $\mathrm{D} 1=100, \mathrm{D} 2=100$ ' are shown in Fig. 15(a), (b), (c), (d), and (e), respectively. Please note that again, LKFS equalization was applied to the spectra. Each simulated data $\mathrm{x}$ denotes the value of a single frequency bin. By comparing the measured spectra with the simulated spectra in Fig. 15 it is confirmed that the top 15 IMD peaks in terms of amplitude appear to match each other well.

To characterize the spectra in terms of significantly large tones of $400 \mathrm{mVp}$-p, the correlations between the measured and simulated peak strengths are plotted in Fig. 16. When the distortion level for even-order nonlinearities is increased with the D2 knob, the second-order peaks of $f_{2}+/-f_{1}$ and their harmonics of $2\left(f_{2}+/-f_{1}\right)$ become significantly larger, and the odd-order peaks of $2 f_{2}+/-f_{1}$ and $2 f_{1}+/-f_{2}$ decrease slightly. The match between the measured and simulated IMD spectra is again fairly good down to approximately $-50 \mathrm{dBV}$, as in the one-tone case.

\subsection{IMD for Consonance}

\subsubsection{Dependence of Consonance IMD on Gain and Starvation: Measurement and Simulation}

Two tones $\mathrm{f}_{1}=369.99\left(\mathrm{~F}_{\# 4}\right)$ and $\mathrm{f}_{2}=554.37\left(\mathrm{C}_{\# 5}\right)$ with a 1:1 amplitude ratio, corresponding to a frequency range similar to that used in the dissonance experiment, were chosen for the consonance IMD measurements. The measured and simulated spectra with distortion knob settings of ' $\mathrm{D} 1$ $=0, \mathrm{D} 2=0,{ }^{\prime} \mathrm{D} 1=0, \mathrm{D} 2=100$, ' 'D1 = 50, D2 = 50 ,' 'D1 $=100, \mathrm{D} 2=0$,' and 'D1 $=100, \mathrm{D} 2=100$ ' are shown in Fig. 17(a), (b), (c), (d), and (e), respectively. The frequency ratio between $\mathrm{F}_{\# 4}$ and $\mathrm{C}_{\# 5}$ is approximately 2:3, yielding a simple spectrum compared with that in the dissonance experiment. The significant new observation is the relatively clean spectrum for the whole frequency range in the case of 'D1 $=100, \mathrm{D} 2=0$,' as seen by comparing Fig. 17(d) with Fig. 15(d). The correlations between the measured and simulated peak strengths are also plotted in Fig. 18. The deviations are smaller than those in the dissonant case.

\subsubsection{Analysis of the Major Spectrum of IMD Peaks in the Case of Consonance}

The fine frequency ratio between $\mathrm{F}_{\# 4}$ and $\mathrm{C}_{\# 5}$ with an equal temperament is 2.000:2.9967, not an integer ratio such as the ratio of 2:3 for a pure temperament. The FFT spectra in the cases of $2 f_{2}-f_{1}$ (cubic difference tone), $f_{2}+f_{1}$, and $3 \mathrm{f}_{2}-\mathrm{f}_{1}$ are shown in panels (a), (a'), (b), (b') (c), and (c') of Fig. 19. The panels on the left-hand side correspond to the ' $\mathrm{D} 1=100, \mathrm{D} 2=0$ ' (odd-rich) setting, and the panels on the right-hand side correspond to the ' $\mathrm{D} 1=100$, $\mathrm{D} 2=100$ ' (even-rich) setting. The frequency resolution employed here is $0.5 \mathrm{~Hz}$, which is an adequate value considering the human-ear frequency sensitivity of $1 \mathrm{~Hz}$, which is called the just-noticeable frequency difference [27] and is also equal to one-quarter of an alternative definition of resolution $(=4 \mathrm{~Hz})$ called the just-noticeable frequency modulation. Therefore by using the higher resolution of 0.5 $\mathrm{Hz}$, the consonance IMD spectrum can be analyzed with and compared to a psychophysical model in which a mechanical simulation is used to model the operation mechanism of the basilar membrane with fine physical resolution [30].

The frequency combinations corresponding to the subpeaks are identified as noted in each panel. It should be noted that the spectra also contain higher-order harmonics, such as $8 \mathrm{f}_{2}-10 \mathrm{f}_{1}$ for the main peak of $2 \mathrm{f}_{2}-\mathrm{f}_{1}, 10 \mathrm{f}_{1}$ $5 f_{2}$ for $f_{2}+f_{1}$, and $9 f_{2}-10 f_{1}$ for $2 f_{1}+f_{2}$. The IMD subtone peaks, which appear at $1 \mathrm{~Hz}$ increments between harmonic peaks, are not harmonics. They are generated by overlap of the skirts of adjacent harmonic peaks due to limited resolution. For example a peak at $738 \mathrm{~Hz}$ is generated by an overlap of harmonic skirts $2 \mathrm{f}_{2}-\mathrm{f}_{1}$ and $4 \mathrm{f}_{2}-4 \mathrm{f}_{1}$ in panel (a).

The distribution of the subpeak heights changes with the distortion knob settings; a sharp-pointed spectrum is obtained for the odd-rich cases of ' $\mathrm{D} 1=100, \mathrm{D} 2=0$ ' shown in panels (a) and (b), while an elliptical cone-shaped spectrum is obtained for the even-rich cases of ' $\mathrm{D} 1=100$, D2 $=100$ ' shown in panels (a'), (b'), and (c').

Fig. 20 shows the same spectra in the extended frequency ranges of $20 \mathrm{~Hz}$ (a), (a'), $30 \mathrm{~Hz}$ (b), (b'), and $40 \mathrm{~Hz}$ (c), (c'). These bandwidths are approximately equal to the unit frequency steps of $\Delta f_{0.2 \mathrm{~mm}}$ defined by a constant step size of $0.2 \mathrm{~mm}$ on the basilar membrane for maximal stimuli of approximately $800 \mathrm{~Hz}, 1 \mathrm{kHz}$, and $1.5 \mathrm{kHz}$, respectively, as defined in [27].

The main property observed in these spectra is symmetry. A clear symmetric peak can be observed in the spectra of $2 \mathrm{f}_{2}-\mathrm{f}_{1}(\mathrm{a})$ and $\mathrm{f}_{2+} \mathrm{f}_{1}$ (b) for ' $\mathrm{D} 1=100, \mathrm{D} 2=0$ ' (rich in odd harmonics). In contrast the spectrum is asymmetric for other cases, with the slope on the higher-frequency side being steeper than that on the opposite side. Additionally the base of the peak in these frequency ranges is approximately -50 $\mathrm{dB}$ from the top of the peak. These features are correctly simulated.

The masking patterns of narrowband sounds have been studied for either sinusoids (added sinusoidal tones) or bands of noise (filtered white noise) in many ways. In early work, it was considered that the masker signal threshold might be directly related to the amount of excitation spreading in the cochlea [31,32]. In general, the slopes of the masking patterns for narrowband noise are very steep on the low-frequency side compared with the high-frequency side [27]. However a variety of systematic differences have been found in the masking shapes between sinusoids and narrowband noise, although they produce similar longterm-average excitation patterns [33-35]. Additionally the thresholds may be influenced by the presence of beats between the signal and masker [36]. Our results suggest that the sound changes associated with IMD for consonance due to even harmonics may be attributable to the spectrum 


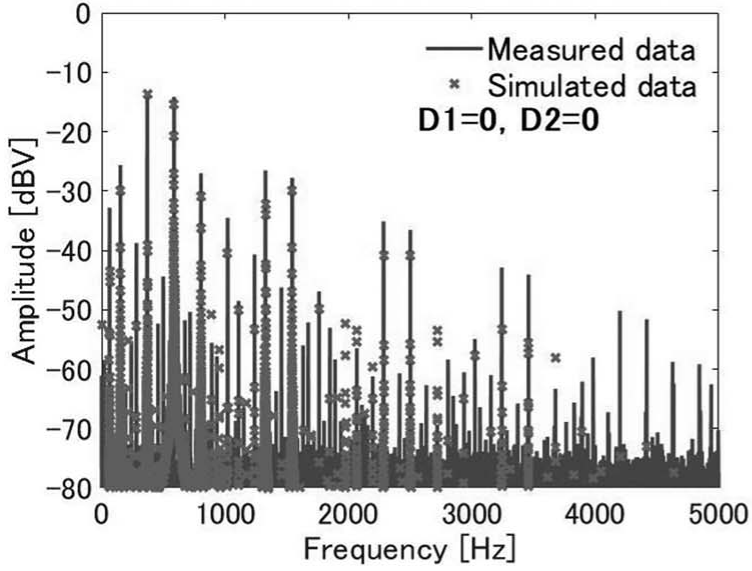

(a)

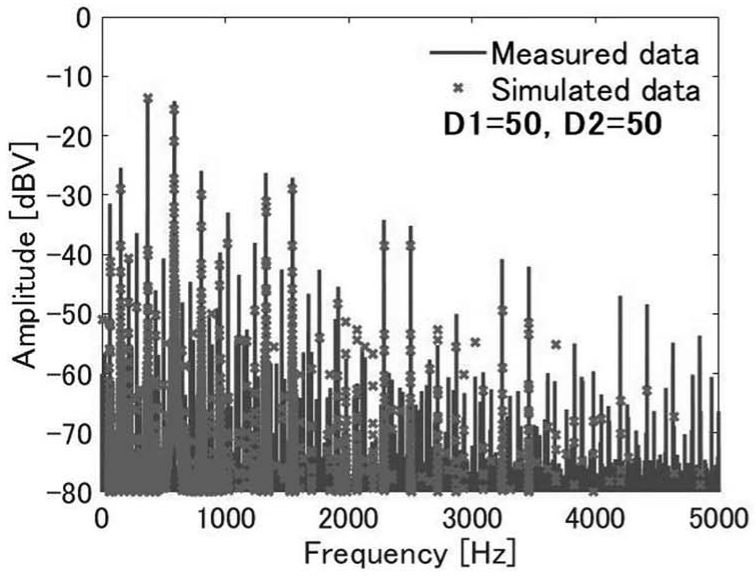

(c)

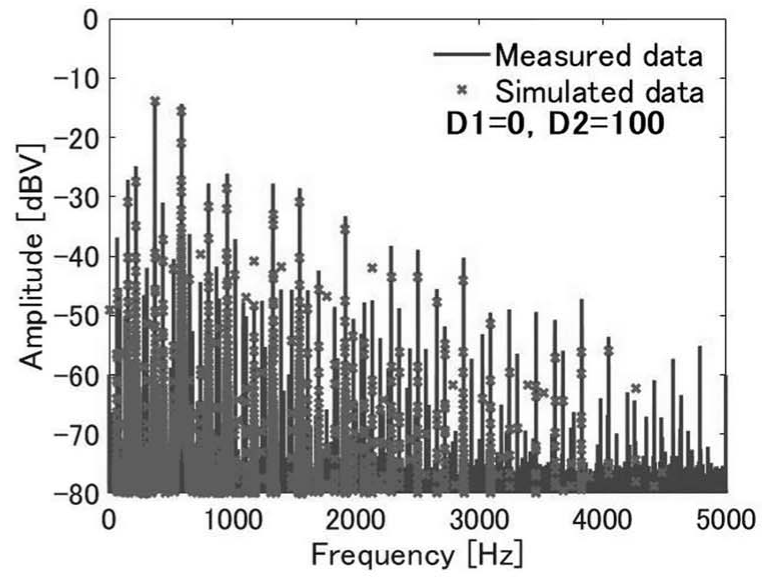

(b)

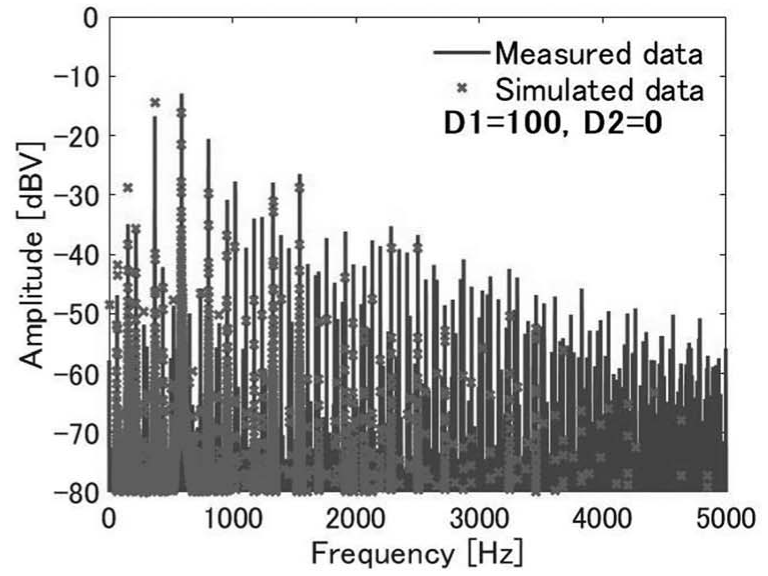

(d)

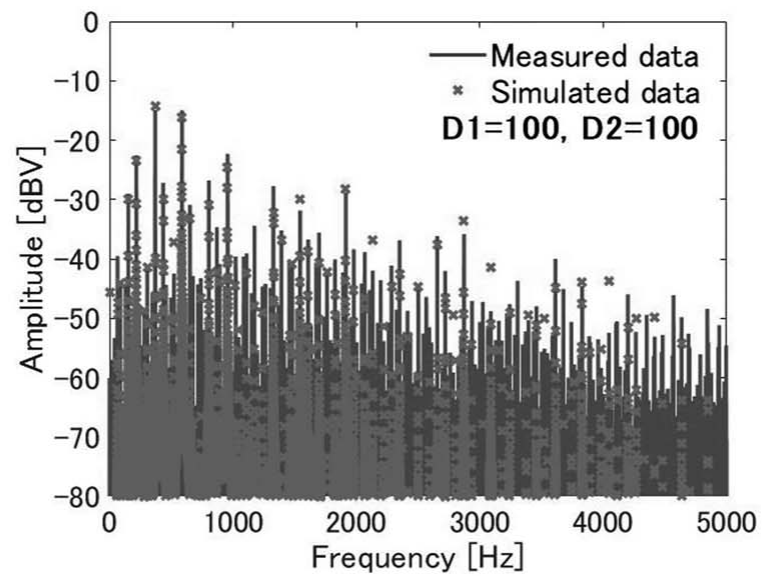

(e)

Fig. 15. Measured and simulated spectra of the distortion pedal output for the input signal $\mathrm{f}_{1}=369.99 \mathrm{~Hz}\left(\mathrm{~F}_{\# 4}\right)$ and $\mathrm{f}_{2}=587.33 \mathrm{~Hz}$ $\left(\mathrm{D}_{5}\right)$, with distortion knob scale settings of 'D1 $=0, \mathrm{D} 2=0,{ }^{\prime} \mathrm{D} 1=0, \mathrm{D} 2=100,{ }^{\prime} \mathrm{D} 1=50, \mathrm{D} 2=50$, ' $\mathrm{D} 1=100, \mathrm{D} 2=0$,' and 'D1 $=100, \mathrm{D} 2=100$ ' shown in (a), (b), (c), (d), and (e), respectively.

shape, depending on the combination of harmonics as well as the strength.

\subsection{IMD Discussion}

In summary, it can be seen from these experimental results on IMD characteristics that the major features of the phase-compensated transfer function, specifically for spectra rich in even and odd-order harmonics, can be reproduced by $9^{\text {th }}$-order polynomial approximations. The IMD peak spectrum in the case of consonance shows an asymmetric shape with a steeper slope on the higherfrequency side, which is simulated well by the transfer function. 


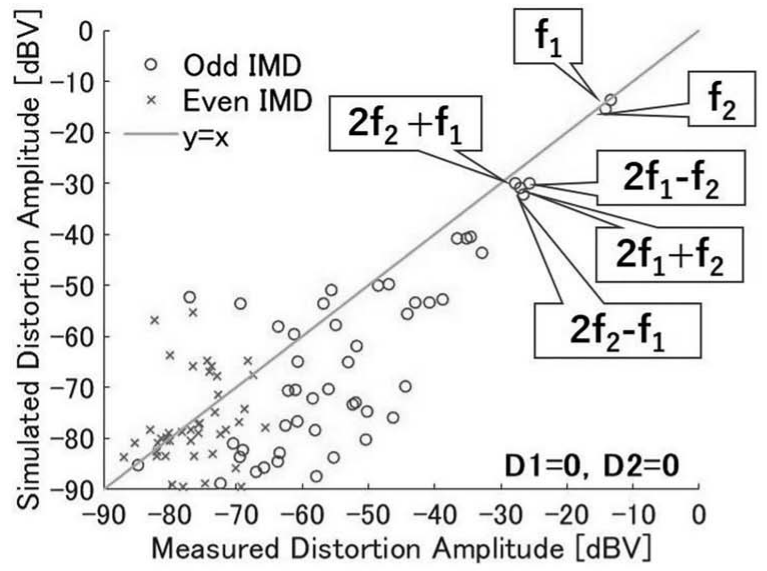

(a)

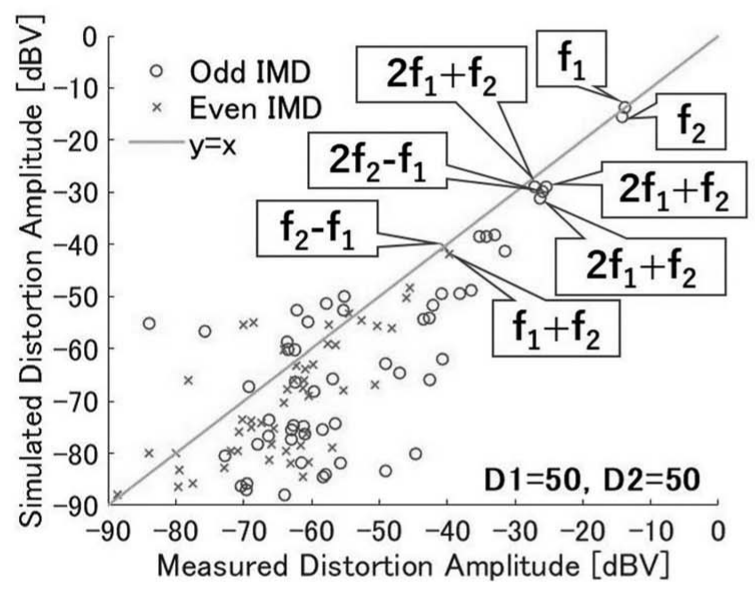

(c)

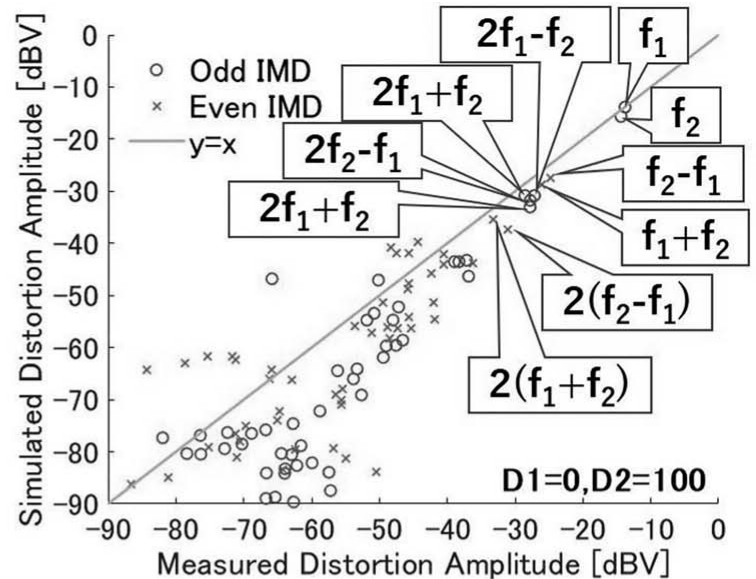

(b)

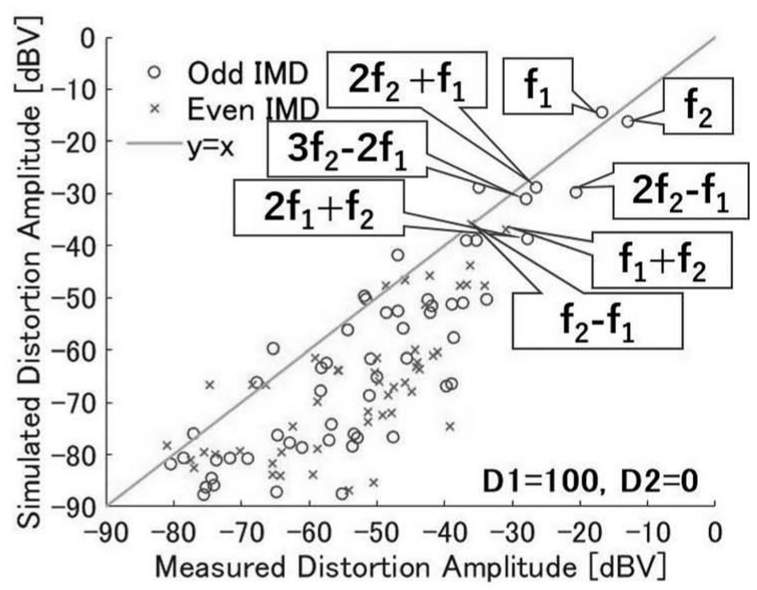

(d)

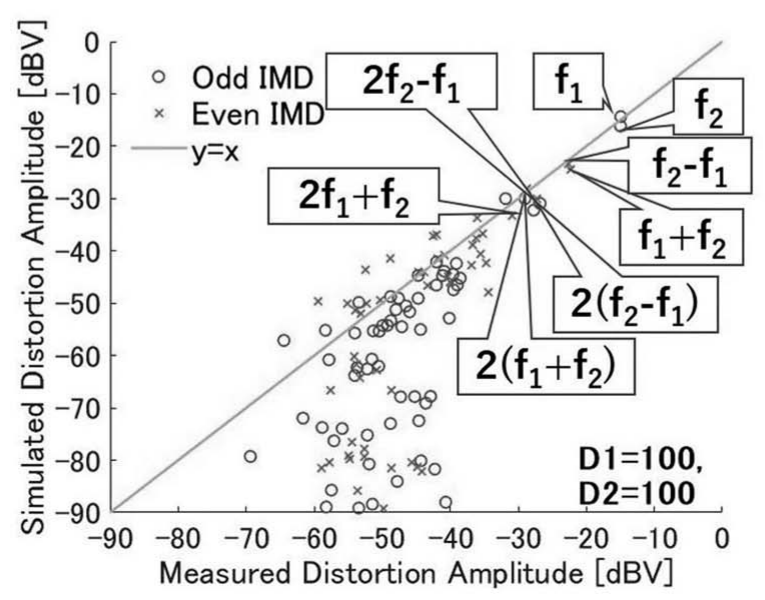

(e)

Fig. 16. Correlation of the strength of tones between the measured and simulated amplitude for the IMD spectra of the input signal $\mathrm{f}_{1}$ $=369.99 \mathrm{~Hz}\left(\mathrm{~F}_{\# 4}\right)$ and $\mathrm{f}_{2}=587.33 \mathrm{~Hz}\left(\mathrm{D}_{5}\right)$, with distortion knob scale settings of ' $\mathrm{D} 1=0, \mathrm{D} 2=0$, ' 'D1 = 0, D2 = 100,' 'D1 = 50, $\mathrm{D} 2=50$, 'D1 = 100, D2 = 0,' and 'D1 = 100, D2 = 100' shown in (a), (b), (c), (d), and (e), respectively. 


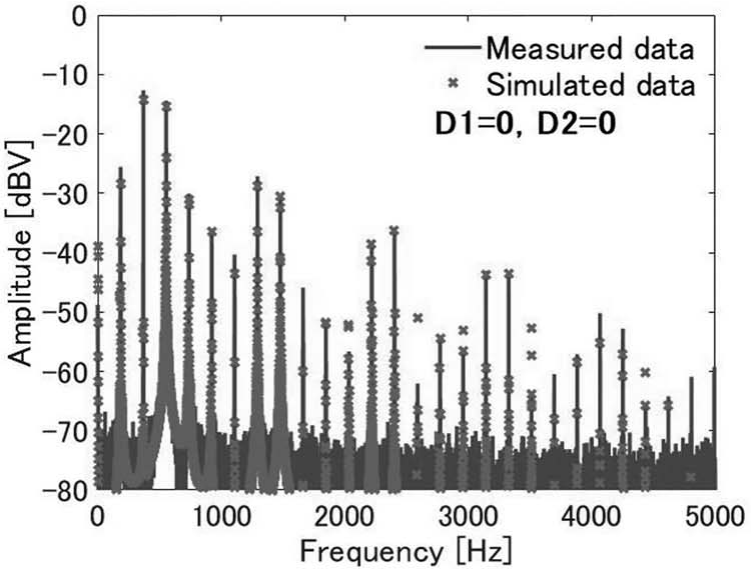

(a)

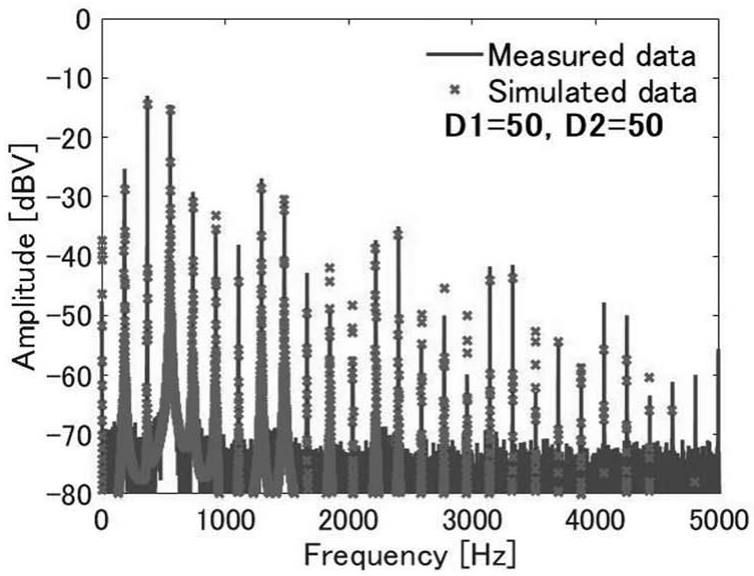

(c)

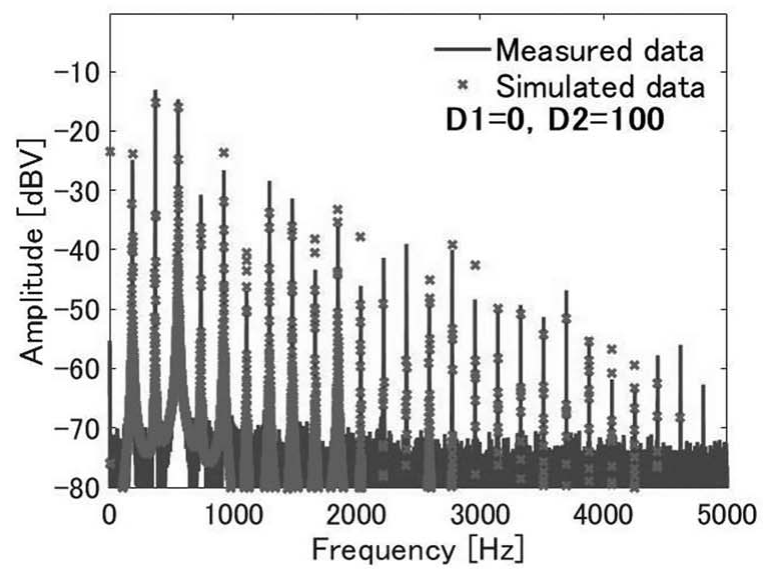

(b)

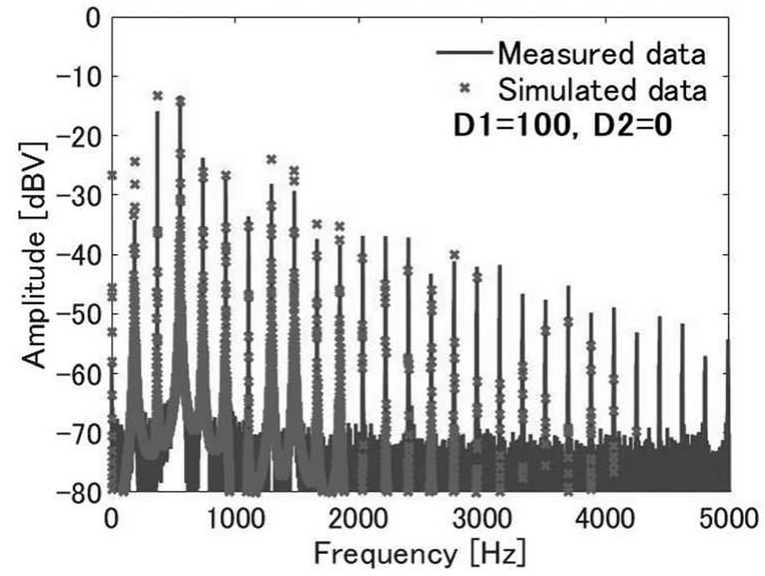

(d)

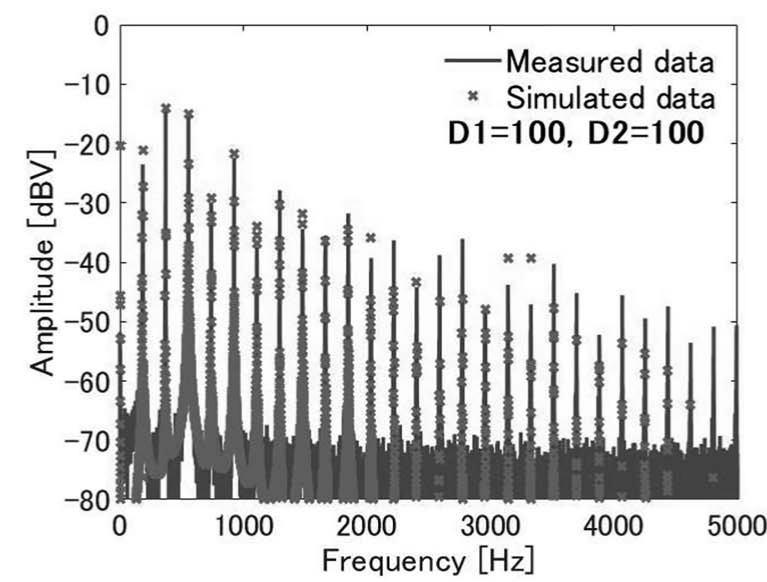

(e)

Fig. 17. Measured and simulated spectra of the distortion pedal output for the input signal $\mathrm{f}_{1}=369.99 \mathrm{~Hz}\left(\mathrm{~F}_{\# 4}\right)$ and $\mathrm{f}_{2}=554.37 \mathrm{~Hz}$ $\left(\mathrm{C}_{\# 5}\right)$, with distortion knob scale settings of ' $\mathrm{D} 1=0, \mathrm{D} 2=0,{ }^{\prime} \mathrm{D} 1=0, \mathrm{D} 2=100$, ' $\mathrm{D} 1=50, \mathrm{D} 2=50,{ }^{\prime} \mathrm{D} 1=100, \mathrm{D} 2=0$,' and 'D1 $=100, \mathrm{D} 2=100$ ' shown in (a), (b), (c), (d), and (e), respectively. 


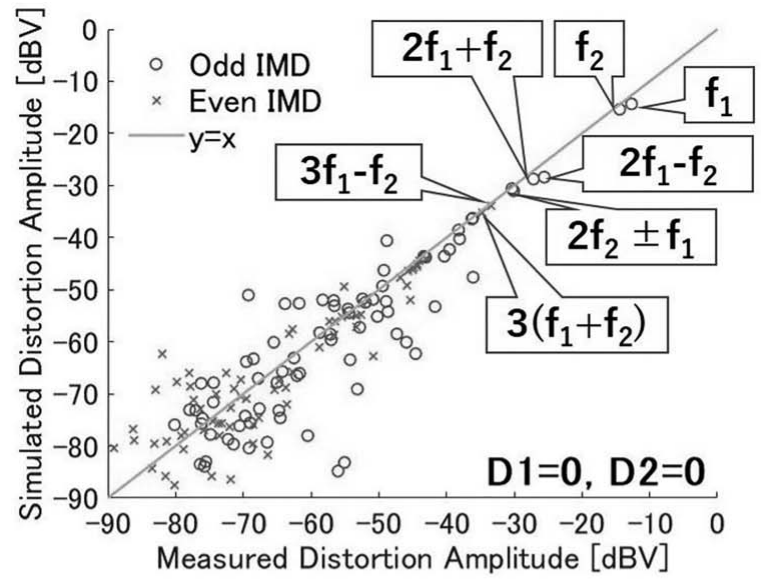

(a)

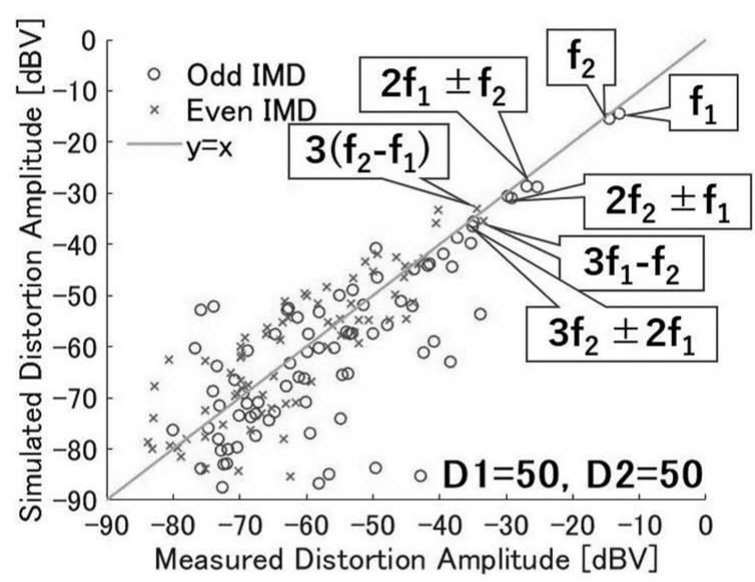

(c)

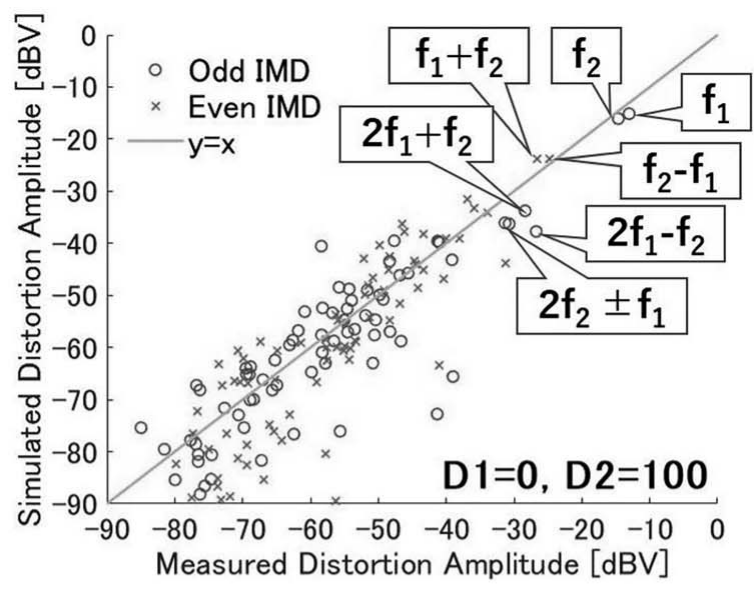

(b)

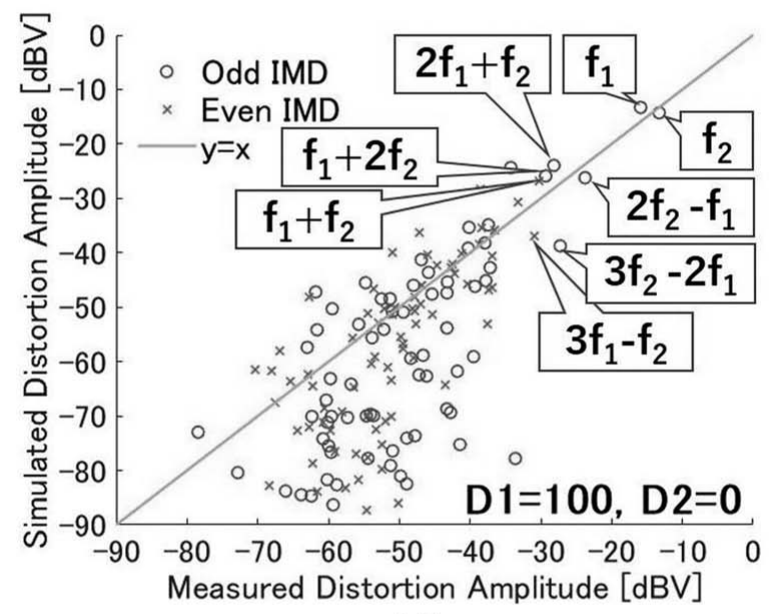

(d)

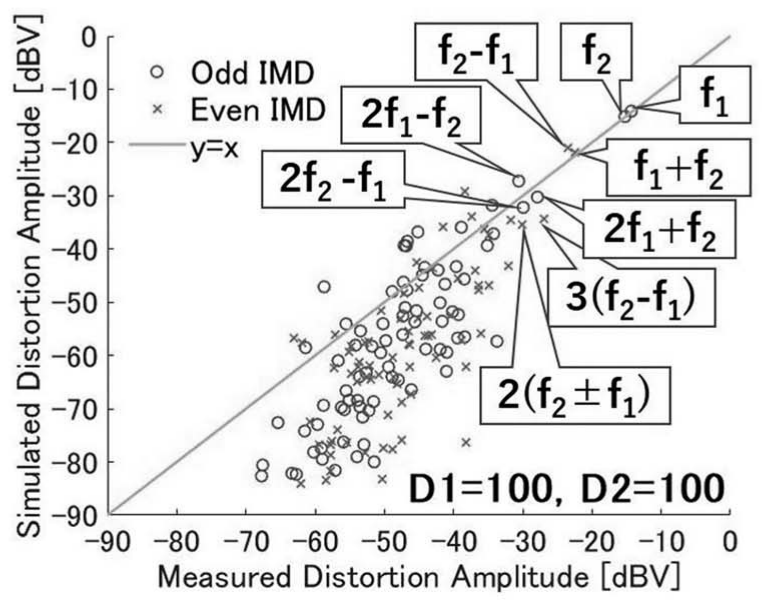

(e)

Fig. 18. Correlation of the strength of tones between the measured and simulated amplitude for the IMD spectra of the input signal $\mathrm{f}_{1}$ $=369.99 \mathrm{~Hz}\left(\mathrm{~F}_{\# 4}\right)$ and $\mathrm{f}_{2}=554.37 \mathrm{~Hz}\left(\mathrm{C}_{\# 5}\right)$, with distortion knob scale settings of ' $\mathrm{D} 1=0, \mathrm{D} 2=0$,' 'D1 = 0, D2 = 100, 'D1 = 50, $\mathrm{D} 2=50$, ' $\mathrm{D} 1=100, \mathrm{D} 2=0$,' and 'D1 = 100, D2 = 100' shown in (a), (b), (c), (d), and (e), respectively. 


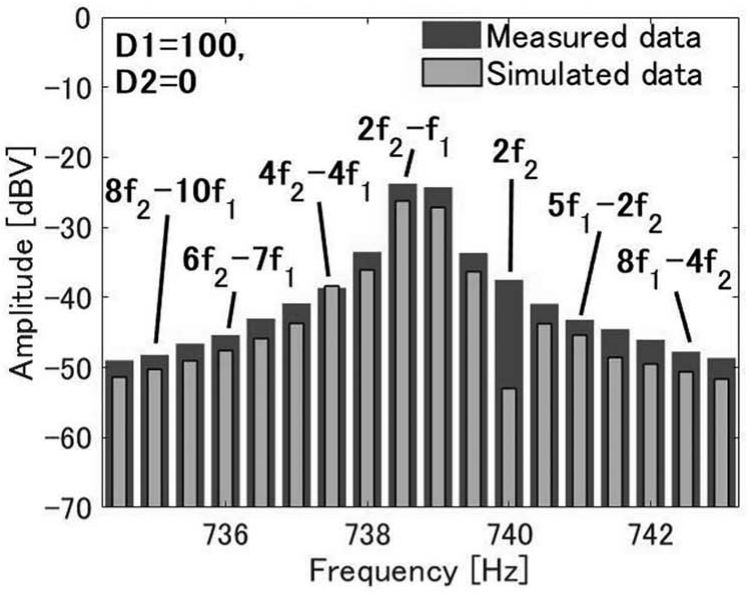

(a)

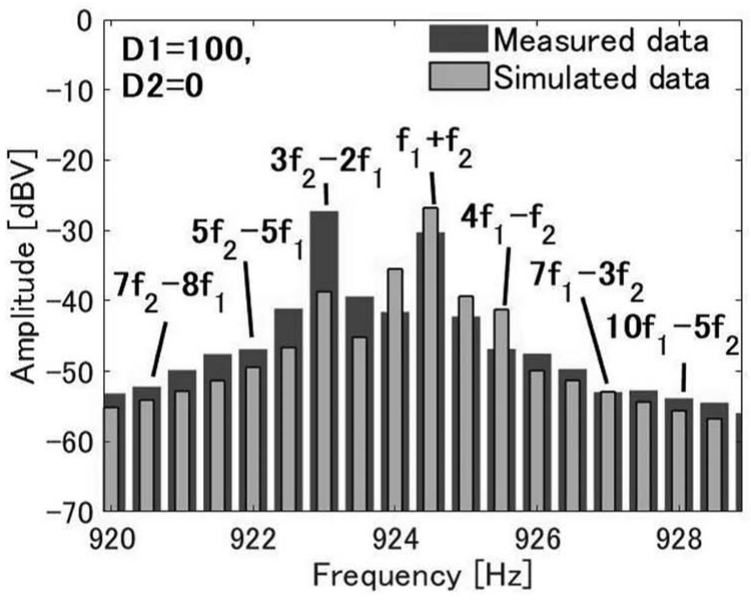

(b)

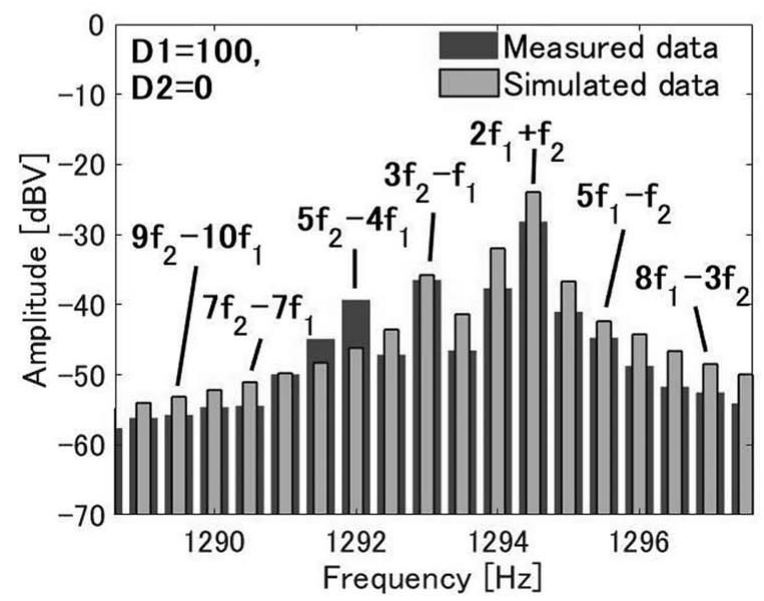

(c)

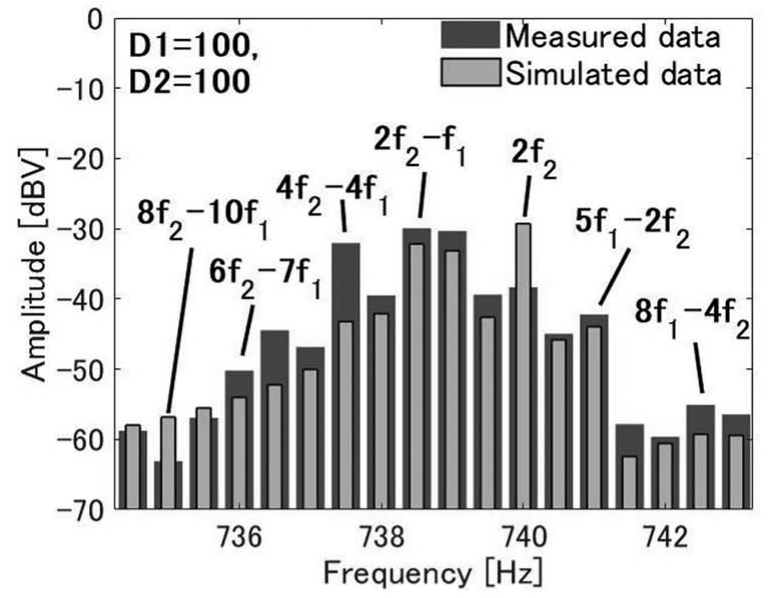

(a')

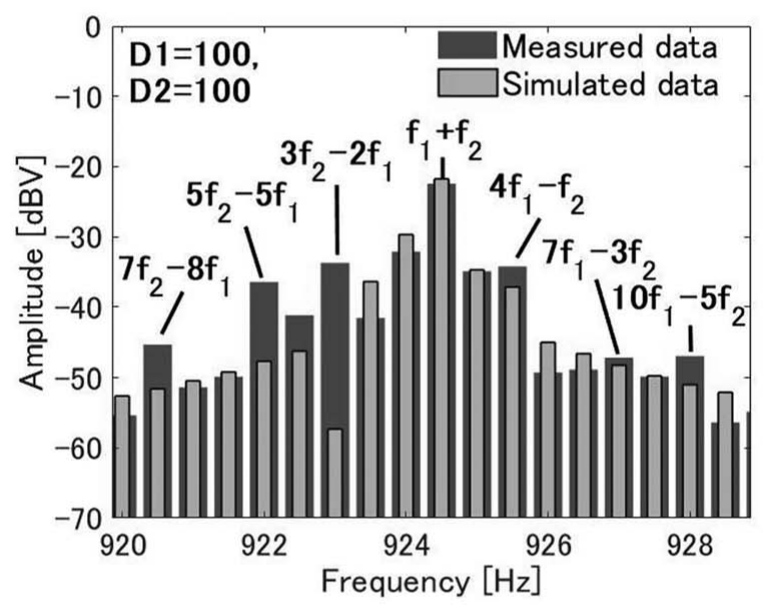

(b')

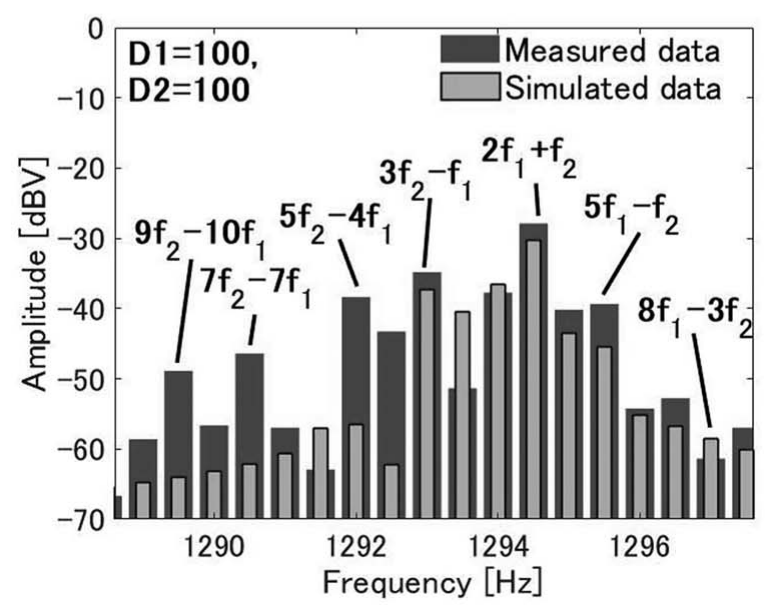

(c')

Fig. 19. Measured and simulated IMD spectra using the transfer function of the distortion knob scale ' $D 1=100, D 2=0$ ': $2 \mathrm{f}_{1}-\mathrm{f}_{2}(\mathrm{a})$, $f_{2}+f_{1}(b)$, and $3 f_{2}-f_{1}(c)$, respectively. ' $D 1=100, D 2=100$ ': $2 f_{1}-f_{2}\left(a^{\prime}\right), f_{2}+f_{1}\left(b^{\prime}\right)$, and $3 f_{2}-f_{1}\left(c^{\prime}\right)$, respectively. 


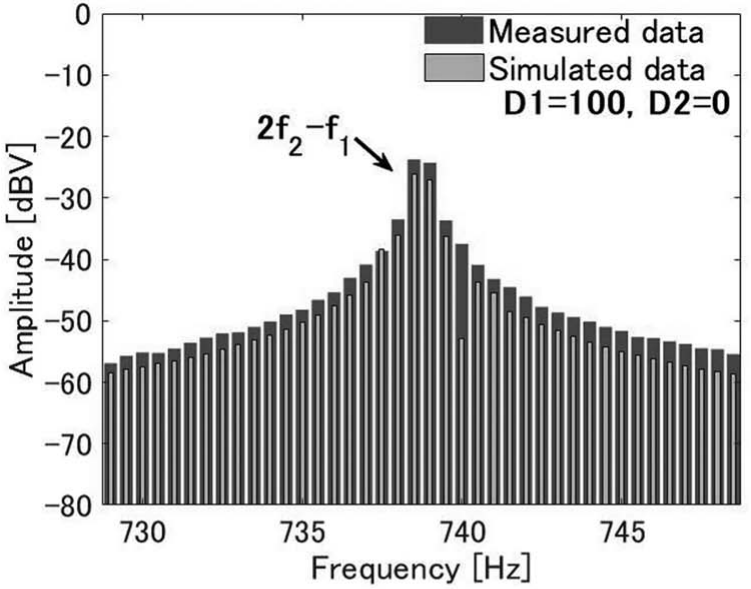

(a)

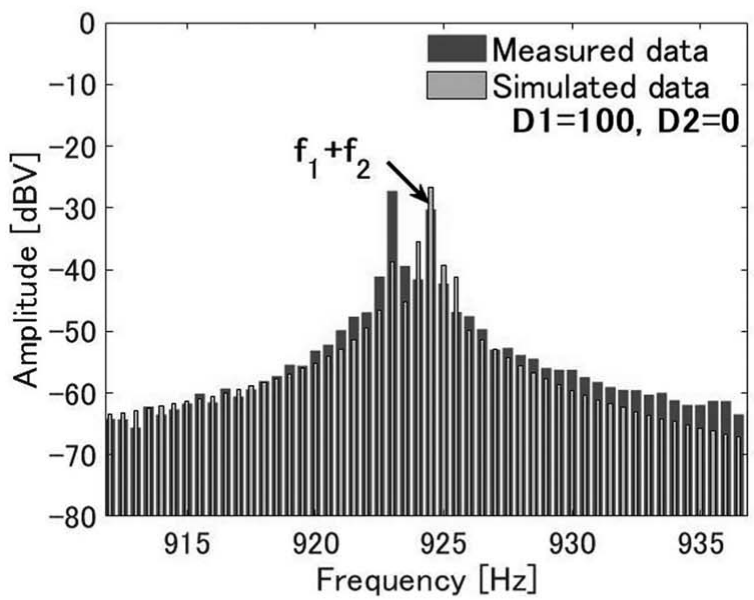

(b)

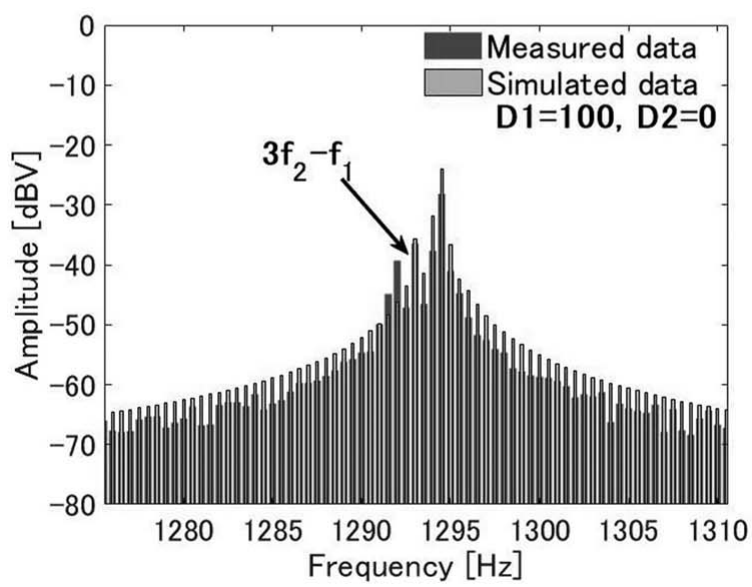

(c)

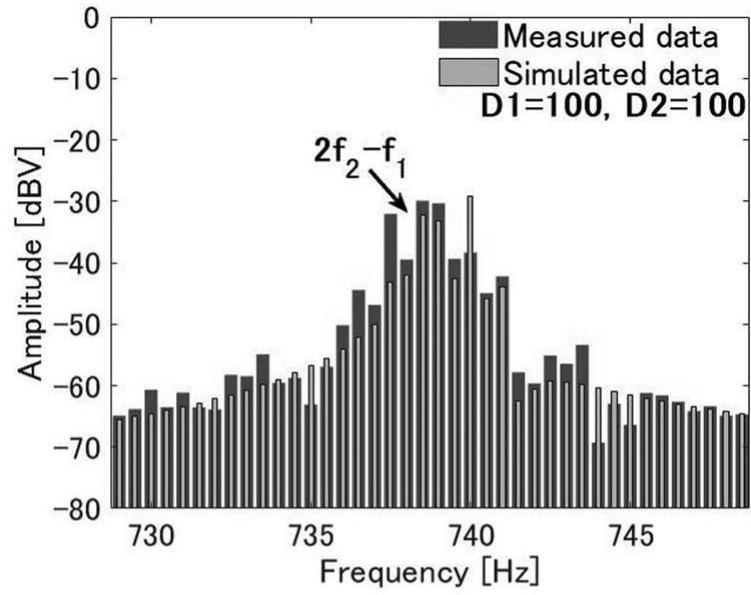

(a')

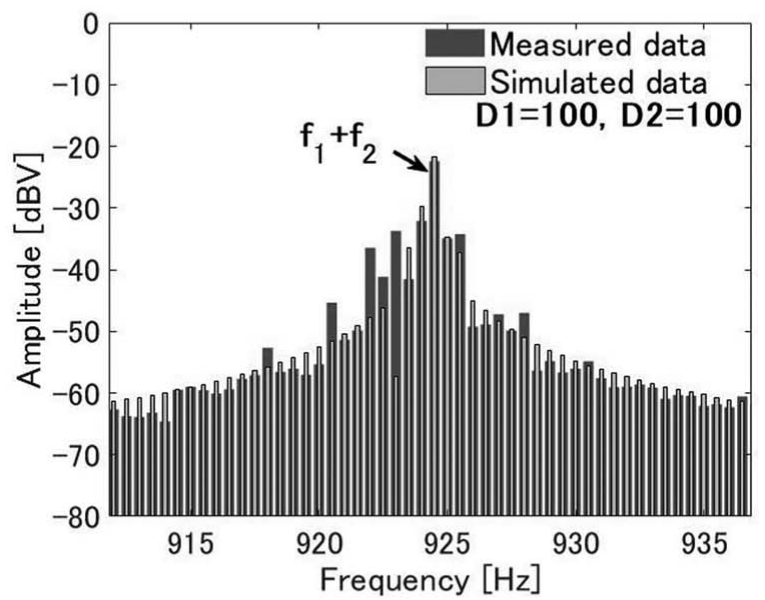

(b')

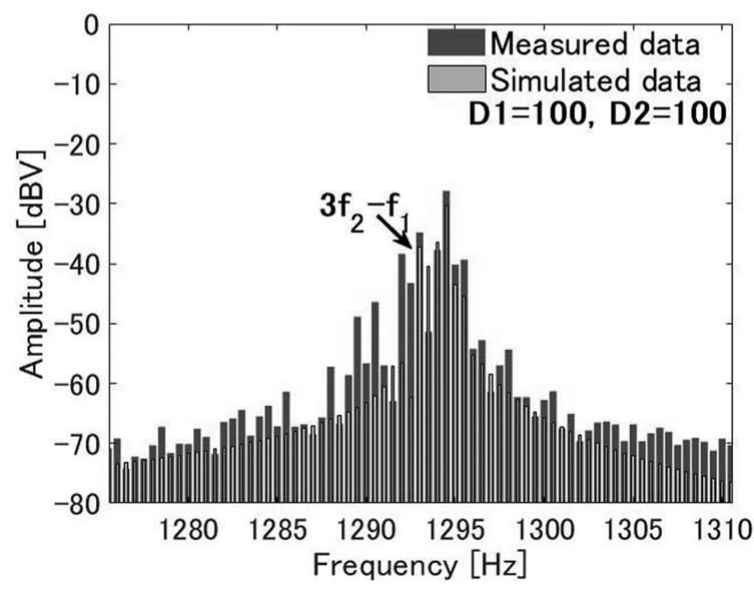

(c')

Fig. 20. Measured and simulated IMD spectra using the transfer function of the distortion knob scale ' $D 1=100, D 2=0$ ': $2 \mathrm{f}_{1}-\mathrm{f}_{2}(\mathrm{a})$, $f_{2}+f_{1}(b)$, and $3 f_{2}-f_{1}(c)$, respectively. ' $D 1=100, D 2=100$ ': $2 f_{1}-f_{2}\left(a^{\prime}\right), f_{2}+f_{1}\left(b^{\prime}\right)$, and $3 f_{2}-f_{1}\left(c^{\prime}\right)$, respectively. 
Therefore a digital implementation with a $9^{\text {th }}$-order polynomial approximation is considered to be applicable even for the dynamic transfer functions to reproduce the nonlinearity of the distortion pedal. However, while the tests conducted show promise for the polynomial modeling technique, perceptual tests still need to be conducted before it can be claimed to be sufficient.

\section{CONCLUSIONS}

It has been clarified that the well-known "voltage starvation" technique for sound change using a distortion pedal is a method for controlling the strength of even-order distortions. This fact has been discovered by investigating the behavior of a newly developed distortion pedal in which the supply voltage of the gain OPA is continuously starved. The effect has been proven by modeling different transfer functions depending on the degree of starvation that are expressed in terms of the superimposition of evenorder and odd-order terms in a $9^{\text {th }}$-order polynomial. Here the error between the measured and simulated spectra is less than $50 \mathrm{~dB}$. Thus, in the case of IMD, we have succeeded in identifying all peak IM frequencies associated with the mathematical formula $\mathrm{nf}_{1}+\mathrm{mf}_{2}$, where $-9<$ $\mathrm{n}<9$ and $-9<\mathrm{m}<9$, in a complex spectrum. Especially for the case of consonance between two tones, it is considered that the asymmetric IMD spectrum of the subpeaks will play the role of a set of maskers to generate the specific sounds associated with voltage starvation. Therefore the $9^{\text {th }}$-order polynomial model is a candidate for an accurate real-time processing method as a virtual analog model of the transfer characteristics of the distortion pedal.

Also it is demonstrated that our new analog distortion pedal is useful for improving statistical perception tests of variations in asymmetric distortion, for which the available conditions used to be limited [2]. In order to confirm its effectiveness we are currently constructing a perceptual evaluation scenario with our new distortion pedal that includes a unit organization for listeners based on the principle of perceptual audio evaluation [37].

\section{REFERENCES}

[1] "1983 Ibanez TS9 Tube Screamer vs 1982 Boss SD-1 Super Overdrive," YouTube (2013 Jul.). https:// www.youtube.com/watch?v=pzua3-xZKHM.

[2] K. Tsumoto, A. Marui and T. Kamekawa, "The Standard Deviation of the Amplitude Spectrum as a Predictor of the Perception of the 'Power' of Distorted Guitar Timbre," presented at the 144th Convention of the Audio Engineering Society (2018 May), convention paper 9771.

[3] K. Tsumoto, A. Marui and T. Kamekawa, "Investigating Factors That Guitar Players to Perceive Depending on Amount of Distortion in Timbre," presented at the 138th Convention of the Audio Engineering Society (2015 May), convention paper 9227.
[4] K. Tsumoto, A. Marui and T. Kamekawa, "Predictors for the Perception of "Wildness" and "Heaviness" in Distorted Guitar Timbre," presented at the 142nd Convention of the Audio Engineering Society (2017 May), convention paper 9748.

[5] W. G. Jung, Audio IC Op-Amp Applications, 3rd ed. (Sams, Indianapolis, IN, 1987).

[6] R. Ghazala, Circuit-Bending: Build Your Own Alien Instruments (Wiley Publishing, Inc., Hoboken, NJ, 2005).

[7] N. Collins, Handmade Electronic Music: The Art of Hardware Hacking, 3rd ed. (Routledge, Abingdon, United Kingdom, 2020).

[8] "How Does DC Voltage Affect Pedal Sound? - That Pedal Show," YouTube (2019 May). https:// www.youtube.com/watch?v=NxH9jK3Ujgk.

[9] D. T. Yeh, J. S. Abel, A. Vladimirescu and J. O. Smith, "Numerical Methods for Simulation of Guitar Distortion Circuits," Comput. Music J., vol. 32, no. 2, pp. 23-42 (2008).

[10] D. T. Yeh, J. S. Abel and J. O. Smith, "Simplified, Physically-Informed Models of Distortion and Overdrive Guitar Effects Pedals," in Proceedings of the 10th International Conference on Digital Audio Effects, pp. 189-196 (2007 Sept.).

[11] K. Dempwolf, M. Holters and U. Zo"lzer, "Discretization of Parametric Analog Circuits for RealTime Simulations," in Proceedings of the 13th International Conference on Digital Audio Effects (DAFx10) (2010 Sept.). http://dafx10.iem.at/proceedings/papers/ DempwolfHoltersZoelzer_DAFx10_P7.pdf.

[12] A. Fettweis, "Wave Digital Filters: Theory and Practice," Proc. IEEE, vol. 74, no. 2, pp. 270-327 (1986 Feb.).

[13] K. J. Werner, W. R. Dunkel, M. Rest, M. J. Olsen and J. O. Smith III, "Wave Digital Filter Modeling of Circuits With Operational Amplifiers," in Proceedings of the European Signal Processing Conference, pp. 1033-1037 (2016).

[14] M. Karjalainen, "Efficient Realization of Wave Digital Components for Physical Modeling and Sound Synthesis," IEEE Trans. Audio, Speech, Language Process., vol. 16, no. 5, pp. 947-956 (2008 Jul.).

[15] K. J. Werner, A. Bernardini, J. O. Smith and A. Sarti, "Modeling Circuits With Arbitrary Topologies and Active Linear Multiports Using Wave Digital Filters," IEEE Trans. Circ. Syst., vol. 65, no. 12, pp. 4233-4246 (2018 Dec.).

[16] F. Esqueda, H. Pöntynen, J. D. Parker and S. Bilbao, "Virtual Analog Models of the Lockhart and Serge Wavefolders," Appl. Sci. vol. 7, no. 12, pp. 1328-1351 (2017 Dec.).

[17] J. D. Parker, F. Esqueda, and A. Bergner, "Modelling of Nonlinear State-Space Systems Using a Deep Neural Network," in Proceedings of the 22nd International Conference on Digital Audio Effects (DAFx-19), pp. 165172 (2019 Sept.).

[18] A. Wright, E.-P. Damskägg, L. Juvela and V. Välimäki, "Real-Time Guitar Amplifier Emulation With 
Deep Learning," Appl. Sci. vol. 10, no. 3, pp. 766-784 (2020 Jan.).

[19] K. Takemoto, S. Oshimo and T. Hamasaki, "An Even-Order Harmonics Control Technique for Analog Pedal Effector," presented at the 143rd Convention of the Audio Engineering Society (2017 Oct.), convention paper 9850.

[20] M. Inui, K. Takemoto and T. Hamasaki, "Harmonics and Intermodulation Distortion Analysis of the EvenOrder Nonlinearity Controlled Effector Pedal," presented at the 144th Convention of the Audio Engineering Society (2018 May), convention paper 9983.

[21] D. Hunter Guitar Effects Pedals: The Practical Handbook, 2nd ed. (Backbeat Books, Milwaukee, WI, 2013).

[22] JRC, "Datasheet NJM4558D" (2019 Feb.).

[23] ITU, "Algorithms to Measure Audio Programme Loudness and True-Peak Audio Level," Recommendation BS.1770-4 (2015 Oct.).

[24] "Orban Loudness Meter for Windows and Mac OS Operating Manual,” (version 2.9.7) (2019 Feb.) https:// www.orban.com/freeorbanloudnessmeter.

[25] MathWorks, "Boundary Effects and the Cone of Influence." https://jp.mathworks.com/help/wavelet/ ug/boundary-effects-and-the-cone-of-influence.html.

[26] H. Yu, K. El-Sankary and E. I. El-Masry, "Distortion Analysis Using Volterra Series and Linearization Technique of Nano-Scale Bulk-Driven CMOS RF Amplifier," IEEE Trans. Circ. Syst., vol. 62, no. 1, pp. 19-28 (2015 Jan.).

[27] H. Fastl and E. Zwicker, Psychoacoustics: Facts and Models, 3rd ed. (Springer-Verlag Berlin Heidelberg, Berlin/Heidelberg, Germany, 2007).
[28] J. Tenney, A History of 'Consonance' and 'Dissonance' (Excelsior Music Publishing Company, New York, NY, 1988).

[29] H. Helmholtz, On the Sensations of Tone as a Physiological Basis for the Theory of Music (1862), trans. from 1877 edition by Alex J. Ellis, p. 194 (Dover, Mineola, NY, 1954).

[30] N. Filipovic, Computational Modeling in Bioengineering and Bioinformatics (Elsevier, Amsterdam, Netherlands, 2020).

[31] R. L. Wegel and C. E. Lane, "The Auditory Masking of One Pure Tone by Another and Its Probable Relation to the Dynamics of the Inner Ear," Phys. Rev., vol. 23, pp. 266-285 (1924 Feb.).

[32] H. Fletcher and W. A. Munson, "Relation Between Loudness and Masking," J. Acoust. Soc. Am., vol. 9, no. 1, pp. 1-10 (1937).

[33] J. P. Egan and H. W. Hake, "On the Masking Pattern of a Simple Auditory Stimulus," J. Acoust. Soc. Am., vol. 22, no. 5, pp. 622-630, (1950).

[34] R. H. Ehmer, "Masking Patterns of Tones," $J$. Acoust. Soc. Am., vol. 31, no. 8, pp. 1115-1120 (1959).

[35] D. D. Greenwood, "Auditory Masking and the Critical Band,” J. Acoust. Soc. Am., vol. 50, pp. 484-502 (1961).

[36] B. C. Moore, J. I. Alcántara and T. Dau, "Masking Patterns for Sinusoidal and Narrow-Band Noise Maskers," J. Acoust. Soc. Am., vol. 104, no. 2, pp. 1023-1038 (1998 Aug.).

[37] S. Bech and N. Zacharov, Perceptual Audio Evaluation - Theory, Method and Application - Theory, Method and Application (Wiley, Hoboken, NJ, 2006). 


\section{THE AUTHORS}

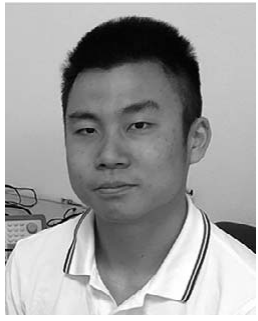

Masaki Inui

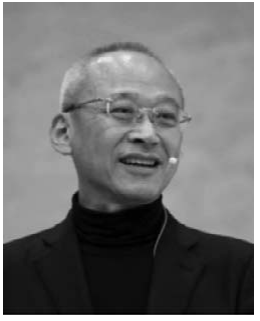

Toshihiko Hamasaki

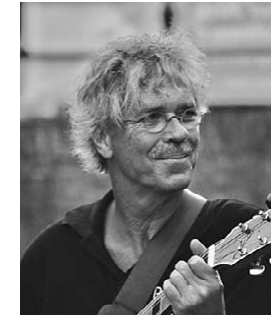

Menno van der Veen
Masaki Inui was born in Hyogo, Japan in 1995. He received a bachelor's degree and master's degree in information science from the Hiroshima Institute of Technology in 2018 and 2020, respectively. He is currently studying for a $\mathrm{Ph} . \mathrm{D}$. at the same university. His research interests are audio signal processing technologies, especially nonlinear characteristics of the analog circuits and systems and instruments as well. He has published conference papers about a guitar effects pedal in AES Conventions 2018 and 2020 and also a couple of papers about a triode tube for audio in the Institute of Electrical Engineers of Japan. Most recently, machine learning of sound characteristics has become one of his research interest areas. He is studying it as an applied method of computer science for dealing with nonlinear phenomena in acoustics. He is a student member of AES.

Toshihiko Hamasaki has been a professor of Information Systems Sciences at the Hiroshima Institute of Technology, Japan since 2010. He received a Ph.D. degree in electrical engineering from Hiroshima University in 1984. In 1984-1991, he worked for the VLSI Research Center in Toshiba Corporation, where he was a researcher of the device physics group. In 1991, he joined Burr-Brown Inc. to design a series of mixed-signal integrated circuits for precision audio DACs, ADCs, and CODECs, covering from high-end professional lines to high-volume consumer lines. In 2001, he moved to Texas Instruments with acquisition and had also been working on analog circuit design of a system on a chip by deep sub-micron advanced technology. A major contribution to AES in his industry career was demonstrations of the world's first development of a high-resolution sigma-delta multi-bit DAC that supports playback of both Super Audio CD and 24-bit/192-kHz PCM formats. On the other hand, his current interests are focused on nonlinear audio systems, especially distorted sound generation. He was awarded TI Fellow in 2004. He is a senior member of IEEE, a senior member of IEICE, and a member of AES.

Menno van der Veen (1949) studied technical physics and electronics at the "Rijks Universiteit Groningen" in the Netherlands. From 1976 to 1990 he taught physics at a high school and a teachers-training college. He worked weekends as a sound engineer in a small alternative theater and was sound coordinator for big outdoor events. Also, as a reviewer, he wrote many articles for Dutch high-end audio magazines, evaluating audio amplifiers. In 1986 he founded his "Vanderveen" company with focus on valve amplifiers with toroidal output transformers. In cooperation with manufacturers worldwide he designed his new range of Vanderveen transformers and regularly reported research results in AES convention papers. He was chairman of the Dutch AES section and co-organizer of AES conventions in the Netherlands. In 2005 he founded a biweekly Saturday school, called "Tube Society," with the goal to share his specific tube amplifier knowledge with enthusiastic do-it-yourselfers. In his private hobby time, he did a lot of research on electronic guitars and their valve amplifiers and effect pedals, almost never publishing research results. Here lies his major origin for high-quality sound reproduction, combined with a love for music and art. This also explains his contribution to the present paper about analog guitar effects pedals. 